\title{
Development and Validation of Quantitative Model-Based Image Reconstruction for NDE of Reinforced Steel-Lined Concrete Structures: Kal-EI FY 2020
}

Hector J. Santos-Villalobos Pradeep Ramuhalli Abdulrahman M. Alanazi

August 2020 


\section{DOCUMENT AVAILABILITY}

Reports produced after January 1, 1996, are generally available free via US Department of Energy (DOE) SciTech Connect.

Website: www.osti.gov/

Reports produced before January 1, 1996, may be purchased by members of the public from the following source:

National Technical Information Service

5285 Port Royal Road

Springfield, VA 22161

Telephone: 703-605-6000 (1-800-553-6847)

TDD: $703-487-4639$

Fax: 703-605-6900

E-mail: info@ntis.gov

Website: http://classic.ntis.gov/

Reports are available to DOE employees, DOE contractors, Energy Technology Data Exchange representatives, and International Nuclear Information System representatives from the following source:

Office of Scientific and Technical Information

PO Box 62

Oak Ridge, TN 37831

Telephone: 865-576-8401

Fax: 865-576-5728

E-mail: report@osti.gov

Website: http://www.osti.gov/contact.html

This report was prepared as an account of work sponsored by an agency of the United States Government. Neither the United States Government nor any agency thereof, nor any of their employees, makes any warranty, express or implied, or assumes any legal liability or responsibility for the accuracy, completeness, or usefulness of any information, apparatus, product, or process disclosed, or represents that its use would not infringe privately owned rights. Reference herein to any specific commercial product, process, or service by trade name, trademark, manufacturer, or otherwise, does not necessarily constitute or imply its endorsement, recommendation, or favoring by the United States Government or any agency thereof. The views and opinions of authors expressed herein do not necessarily state or reflect those of the United States Government or any agency thereof. 


\section{Cyber and Applied Data Analytics Division}

\section{Development and Validation of Quantitative Model-Based Image Reconstruction for NDE of Reinforced Steel-Lined Concrete Structures: \\ Kal-EI FY 2020}

Hector J. Santos-Villalobos, Pradeep Ramuhalli, and Abdulrahman M. Alanazi

Date Published: August 2020

Prepared by

OAK RIDGE NATIONAL LABORATORY

Oak Ridge, TN 37831-6283

managed by

UT-Battelle, LLC

for the

US DEPARTMENT OF ENERGY

under contract DE-AC05-00OR22725 



\section{CONTENTS}

LIST OF FIGURES $\ldots \ldots \ldots \ldots \ldots \ldots \ldots \ldots \ldots \ldots$

LIST OF TABLES $\ldots \ldots \ldots \ldots \ldots \ldots \ldots \ldots \ldots$ vii

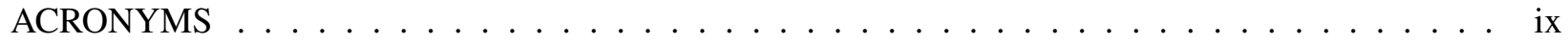

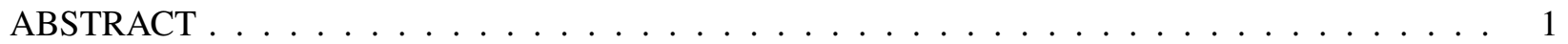

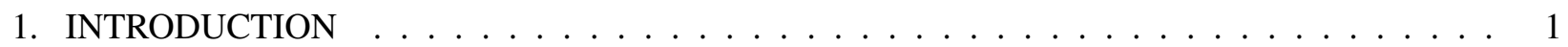

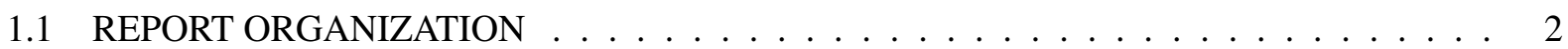

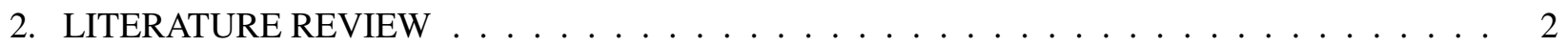

3. SPECIMEN SIMULATION $\ldots \ldots \ldots \ldots \ldots \ldots \ldots \ldots$

3.1 SIMULATION SETUP $\ldots \ldots \ldots \ldots \ldots \ldots \ldots \ldots$

3.1 .1 Simulation Geometry . . . . . . . . . . . . . . . . . . . . . 4

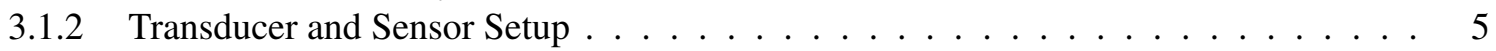

3.1 .3 Material Properties . . . . . . . . . . . . . . . . . . 5

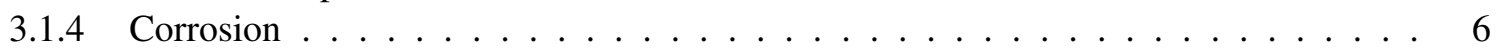

3.1.5 Simulation Overview and Limitations $\ldots \ldots \ldots \ldots \ldots$

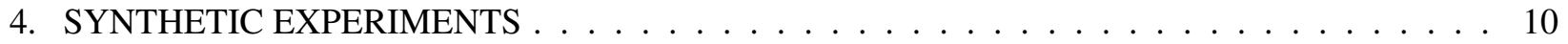

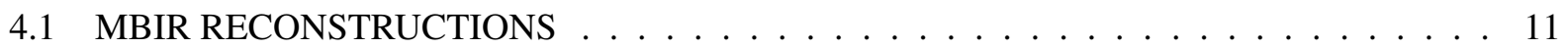

4.1 .1 MBIR Configuration . . . . . . . . . . . . . . . . . . 13

4.1 .2 Average Intensity Measure . . . . . . . . . . . . . . . . . . . 15

4.1.3 Reconstruction Results . . . . . . . . . . . . . . . . . . . . . 17

4.1.4 Reconstruction Results with Baseline Cancellation Method . . . . . . . . . . . . . . 19

4.2 CORROSION CLASSIFICATION VIA MACHINE LEARNING . . . . . . . . . . . . 22

4.2 .1 Ultrasound Samples Extraction . . . . . . . . . . . . . . . . . . . 23

4.2.2 Feature Engineering and Extraction and ML Model Training . . . . . . . . . . . . 25

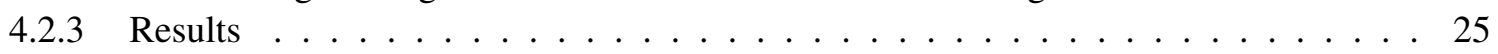

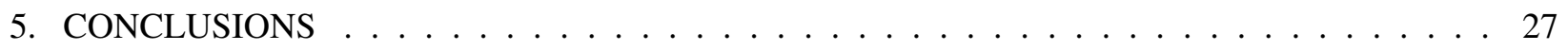

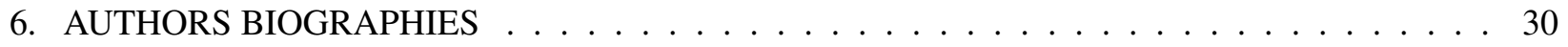

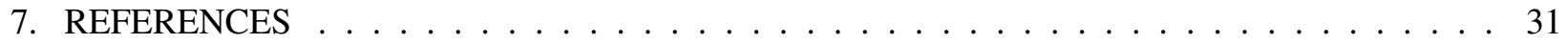





\section{LIST OF FIGURES}

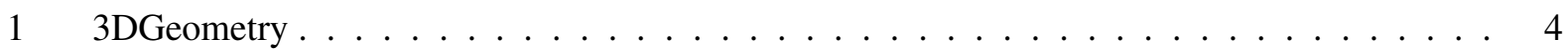

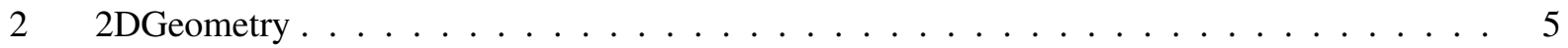

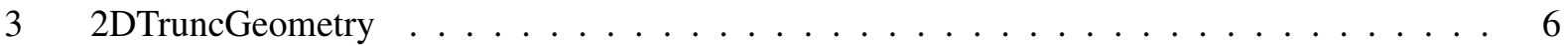

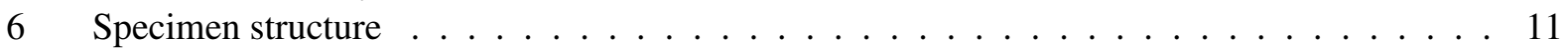

7 An illustration of a typical ultrasonic one-sided flight path diagram. $s(t)$ is the transmitted signal, $v$ is a point in the field of view, $y_{i, j}(v, t)$ is the received signal reflected from $v, \theta_{t}$ is the angle between $r_{i}$ and $v$, and $\theta_{r}$ is the angle between $r_{j}$ and $v_{\ldots} \ldots \ldots \ldots \ldots \ldots$

8 Examples of image reconstructions for different MBIR image pixel sizes. . . . . . . . . 13

9 Examples of image reconstructions for extreme MBIR regularization parameters. . . . . . . 14

10 MBIR reconstructions of simulated data . . . . . . . . . . . . . . . . . 16

11 MBIR reconstructions of K-wave synthetic data . . . . . . . . . . . . . . 17

12 Reconstructed images average pixel intensity distributions per corrosion level. . . . . . . . 19

13 Baseline cancellation illustration. The left image is the original signal and the right image is the signal on the left after baseline cancellation. . . . . . . . . . . . . . 20

14 MBIR-BCM reconstructions of K-wave synthetic data . . . . . . . . . . . . . . . 21

15 MBIR-BCM method reconstructed images average pixel intensity distributions per corrosion level. . . . . . . . . . . . . . . . . . . . . . . . . . 22

16 Illustration of wave flight path from source $k$, to rebar, to receiver $j \ldots \ldots \ldots 23$

17 Illustration of data extraction from original signal: blue line is the original signal, the right and left vertical lines are the lower and upper bounds for the extraction window, the middle vertical line is the estimated time for the ultrasound wave to reach the rebar's center, and the purple line is the extracted signal. . . . . . . . . . . . . . . . . 24

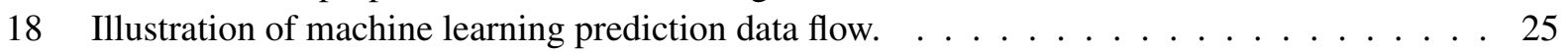

19 Confusion matrix for Per-Signal Model. . . . . . . . . . . . . . . . . . 26

20 Confusion matrix for Per-Scan (a) Average-fusion and (b) Median-fusion methods. . . . . . 27 



\section{LIST OF TABLES}

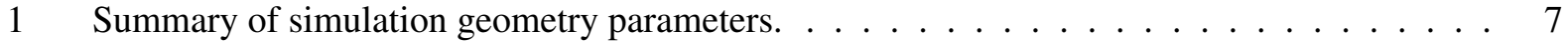

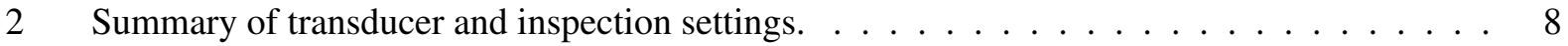

3 Material properties used in k-Wave simulations. . . . . . . . . . . . . . . 9

$4 \quad$ MBIR parameter settings used in K-wave data reconstructions. . . . . . . . . . . . . . . . 14

5 Summary of AIM scores for images in Figure $11(\epsilon=0.1) \ldots \ldots \ldots \ldots$

6 Summary of AIM scores for images in Figure $14(\epsilon=0.1) \ldots \ldots \ldots$. . . . . . . . 22

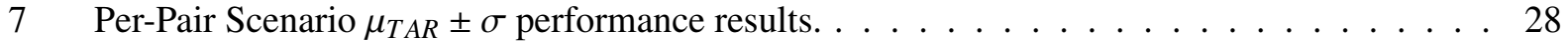





\section{ACRONYMS}

AI artificial intelligence

AIM average intensity measure

$\mathrm{Hz} \quad$ hertz

MBIR model-based image reconstruction

ML machine learning

mm millimeters

NDE nondestructive evaluation

ORNL Oak Ridge national laboratory

PML perfectly matched layer

ROI region of interest

SAFT synthetic aperture focusing technique

TAR true acceptance rate

XGBoost scalable, portable and distributed gradient boosting library 



\begin{abstract}
This project studied the capabilities of Model-Based Image Reconstruction (MBIR) and Machine Learning (ML) algorithms in the imaging and estimation of rebar corrosion from ultrasound signals. The application was challenging due to the introduction of a steel liner between the sensor and the concrete containing the rebar. The study focused in a synthetic specimen with a $6.35 \mathrm{~mm}$ thick steel liner, and a $19.05 \mathrm{~mm}$ diameter rebar at a depth of $44.5 \mathrm{~mm}$ and with four levels of corrosion; 0\%, 20\%, 50\%, and 100\% corrosion level. The corrosion was applied uniformly around the rebar, generating a ring in the perimeter of the rebar with different acoustic density. In order to generate a realistic synthetic dataset, we added random texture to the concrete of the synthetic specimens. This texture will mimic the variations encountered in real concrete specimens.

We adapted our MBIR algorithm for the application and enhanced the algorithm to integrate in the physical model known specimen features, such as the steel liner thickness. The new MBIR method was able to cancel out reverberation from the steel liner and properly image the rebar. In particular, corrosion for the $50 \%$ and $100 \%$ cases were easily visible and quantitative metrics showed a slight separation for the $0 \%$ and $20 \%$ levels. The quantitative results were in agreement with the qualitative assessment. We also developed a ML XGBoost model to estimate corrosion level from the ultrasound signals. By combining the ML method with MBIR, we can pinpoint in the ultrasound signals the section that corresponds to the echoes from the rebar. The extract signals are processed by the XGBoost model for prediction. From each system scan, we obtain 15 predictions. The median prediction value is used as the final prediction. The True Acceptance Rate for the method is over $99 \%$.
\end{abstract}

\title{
1. INTRODUCTION
}

This project studied the capabilities of ultrasonic NDE methods, by using nonlinear elastic wave propagation simulations and model-based image reconstruction (MBIR). The sub-objective of this simulation study was to develop an ultrasonic inspection computational model for the purpose of obtaining synthetic ultrasonic NDE responses from corroded rebars embedded within concrete slabs. The complexity of the model is enhanced by adding a steel liner layer between the ultrasound system and the concrete slab. The simulated signals are intended for use in developing a MBIR and machine learning (ML) algorithms for identifying the presence of corrosion in rebars.

Non-destructive evaluation of thick, solid specimens is a challenge with unknown complete solutions. Usually, solutions trade between resolution and penetration and design revolve around specific features or defects. The challenge compounds when access to the specimen is one-sided and the specimen is layered with large differences in acoustic impedance. This description accurately describe the imaging conditions for the application of interest.

For this project, we attempt at developing quantitative techniques for rebar corrosion inside a reinforced concrete specimen with a frontal steel liner. As explained before, this is a challenging configuration for imaging and even simple measurements. Given the desired range and resolution, we selected low nominal frequency ultrasound imaging as the modality for this application. Given that a solution for this application is non-existent, we scoped this project to simulated ultrasound data.

Through this study, we attempt to answer the following questions: 
- Is off-the-shelve MBIR powerful enough to resolve corrosion from NDE signals?

- Can we distinguish corrosion level from MBIR reconstructions alone?

- If some parts of the specimen are well known, can MBIR produce more accurate reconstructions?

- Can we develop complementary machine learning techniques to estimate corrosion level?

- What are the technological gaps and requirements to develop an actual inspection system?

\subsection{REPORT ORGANIZATION}

This technical report is organized as follows. First, we provide in Section 2. a review of previous work in the fields of ultrasound reconstruction and corrosion detection. That is followed in Section 1.1 with a description of the ultrasound instrument and specimen for this study, which includes their geometry and acoustic properties, and for the specimen the embedded features simulated. This section also includes our approach in the simulation of corrosion. We finalize the section documenting the employed simulation engine and its limitations. Section 4. gives an overview of the simulations, the MBIR algorithm and our machine learning corrosion detection model. Subsection 4.1 discusses the MBIR algorithm and the specific parameters for this particular implementation, a new metric for quantitative assessment of the reconstruction and the reconstruction results. Then, Subsection 4.2 discusses our machine learning approach for corrosion estimation and document the experimental results. Finally, we end the report with a conclusions section in Section 4.2.3 and also in the last two sections we provide a brief background on the authors and the report references.

\section{LITERATURE REVIEW}

NDE with ultrasound signals is widely employed because of its low cost, high penetration, portability, and safety compared with other NDE methods Ramuhalli et al. (2012); Berndt (2001); Zemanek et al. (1970). A typical ultrasound system will have one or multiple sources and receivers coupled to the specimen. For

one-sided specimens, the receivers measures the reflected signals or echoes. Ultrasound imaging is used on a variety of applications and use cases Hoegh and Khazanovich (2015); Stepinski (2007).

The challenges for ultrasound imaging are signal loss between the transducers and the specimen interface, complex physics for wave propagation, accurate modeling of the instrument, and the trade-off between resolution and penetration Li and Hayward (2011); Haldorsen et al. (2006). Synthetic aperture focusing technique (SAFT) is the most popular method for ultrasound reconstruction, because it is not computationally demanding making its implementation on mobile platforms simpleShao et al. (2011); Engle et al. (2014); Dobie et al. (2013); Beniwal and Ganguli (2015); Hoegh and Khazanovich (2015); Schickert et al. (2003). However, SAFT is a delay and sum approach and prone to reverberation and ghost artifacts Beniwal and Ganguli (2015); Schickert et al. (2003).

There are alternate methods to SAFT that employ regularized iterative reconstruction with linear modelsAlmansouri et al. (2018a); Tuysuzoglu et al. (2012); Ozkan et al. (2017); Wu et al. (2015); Guarneri et al. (2015). These methods formulate the reconstruction as minimizing a cost-function that balances a data fidelity term with a regularization applied to the object to be reconstructed. The data fidelity term contains the physics that maps the object to the measurement space. 
The use of NDE for corrosion detection in engineering alloys is widespread Agarwala et al. (2000); Singh et al. (2019), with visual, magnetic flux leakage, eddy current, and ultrasonic NDE being among the common methods employed for this purpose. Variants of these methods are sometimes used for specific purposes, such as detecting and monitoring corrosion-assisted cracking Singh et al. (2019). While these methods are relatively reliable for detecting corrosion and corrosion-assisted cracking, many of them are challenged when applied to detect hidden corrosion Agarwala et al. (2000).

The issue of hidden corrosion arises in the specific context of corroded rebars embedded in reinforced concrete, with no direct access to the rebars for inspection or monitoring. The problem of corrosion detection can be further exacerbated in steel-concrete composites, where the presence of a steel liner on one or multiple surfaces of the concrete can inhibit the application of many of the NDE techniques that may be relevant Maack et al. (2015); Villalobos et al. (2019). Consequently, the problem of rebar corrosion detection in reinforced concrete has seen a significant amount of research. Conventional NDE inspection solutions that have been proposed include acoustic emission Ramadan et al. (2008), capacitance-based measurements Cheng et al. (2018), eddy current de Alcantara Jr. et al. (2016), microwaves and GPR Pieper et al. (2014), ultrasonics (measuring linear or non-linear response, or using ultrasonic guided waves) Beard et al. (2003); Yeih and Huang (1998); Watanabe et al. (2014); Sriramadasu et al. (2019); Singh et al. (2019). Monitoring methods using embedded fiber-optic sensors have also been proposed Zou et al. (2015), though these are limited in that they are not applicable to structures that were built without any embedded fiber.

The conventional NDE methods that have been proposed are also often difficult to apply for monitoring steel-concrete composites, as the presence of the steel liner acts as a shield for most electromagnetic methods (capacitive, eddy current, microwave/GPR) with magnetic flux leakage methods likely providing some (limited) sensitivity to rebars that are very close to the steel-concrete interface. The sensitivity of ultrasonic guided waves is going to depend on the mode of inspection, where the highest sensitivity will be from directly injecting the ultrasonic energy into the rebar at one end Beard et al. (2003); Li et al. (2014). Obviously, this is going to require access to individual rebars and may not always be an option. Bulk ultrasonic inspection methods provide an alternative method of interrogating rebars by examining ultrasonic energy reflected and scattered from rebars to detect the presence of corrosion. Such methods depend on two factors - the ability to apply a sufficient amount of energy to the material to enable interaction with rebars that may be deep in the concrete, and a difference in the acoustic impedance of rebars with and without corrosion Tang and Yu (2017). The amount of energy that can be applied to the concrete from the surface (steel) is a function of the acoustic impedance mismatch at the interface between the steel and concrete and influenced by delamination of the steel from concrete or the presence of water in the concrete. The acoustic impedance of rebars is a function of the acoustic sound speed, and has been shown to differ between rebars with and without corrosion François et al. (2012, 2018a).

These considerations point to the potential for applying ultrasonic measurements to detect corrosion in rebars, though this has to be verified through a combination of simulation and experimental studies. A critical aspect of this assessment is the ability to use image reconstruction methods to detect the presence of corrosion. This report describes the result of applying model-based image reconstruction to simulation studies to determine the viability of using ultrasonic NDE for detecting corrosion in rebars in reinforced steel-lined concrete structures. 


\section{SPECIMEN SIMULATION}

\subsection{SIMULATION SETUP}

\subsubsection{Simulation Geometry}

The ultrasound wave simulation software employed for this study is k-Wave (Treeby and Cox (2010); Treeby et al. $(2012,2014,2018))$. k-Wave is an open source library implemented in MATLAB and the C language for acoustic, photoacoustic, and elastic wave propagation simulation. The simulator can handle 2D and 3D simulations of homogeneous or heterogeneous specimens. Therefore, this software package was found fit for this exploratory phase to study the performance of MBIR and ML algorithms in the detection and measurement of corrosion inside a concrete specimen behind a steel liner.

Simulations are performed using a 2D geometry, consisting of a slice in the $\mathrm{x}-\mathrm{y}$ plane of a 3D concrete structure with a steel liner. Figure 1 shows the geometry in 3D of the full specimen while Figure 2 shows a 2D slice. Figure 2 also shows a headed stud anchor in the concrete. For the current simulation runs, the anchor is not included in the simulations. After initial simulation of the full specimen, we quickly noted that imaging of the back rebar was unattainable from a real system, while imaging of the front rebar was achievable. Therefore, we decided to limit simulation of the specimen to the steel liner and 4 in. of concrete slab Figure 3. This section of the slab includes the rebar closest to the steel liner. A perfectly matched layer (PML) was added around the structure to eliminate reflections from the interface between the concrete slab and air. The PML layer simulates that the specimen extends infinitely at the edges.

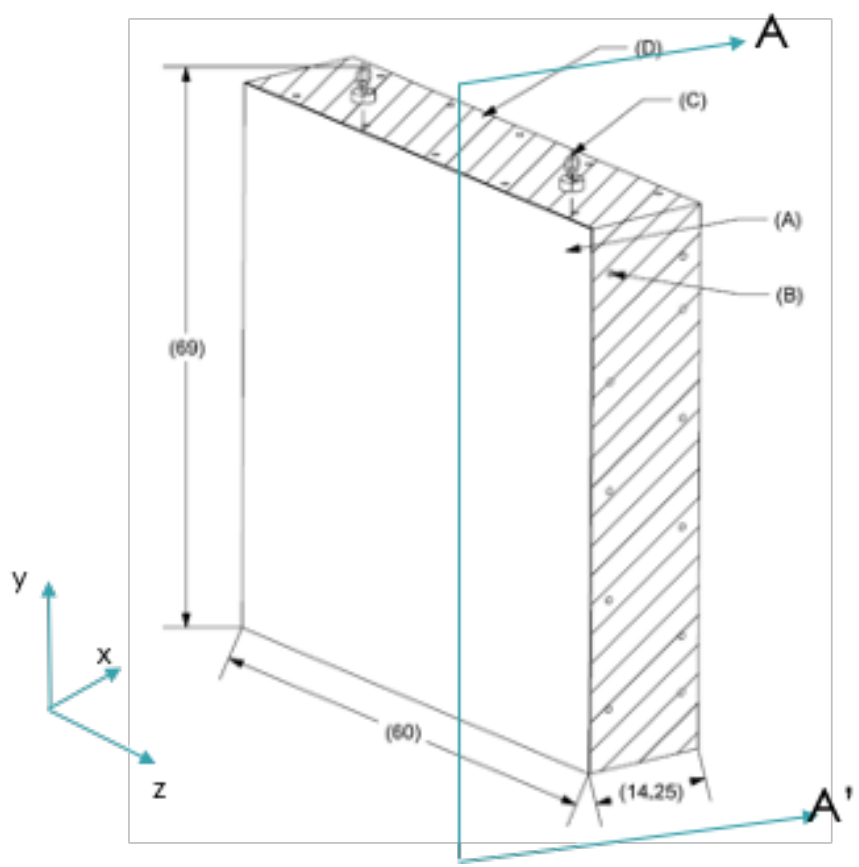

Figure 1. 3D geometry of concrete slab with rebars and steel liner.

Details of the geometry (dimensions) used in the simulation are in table 1. 


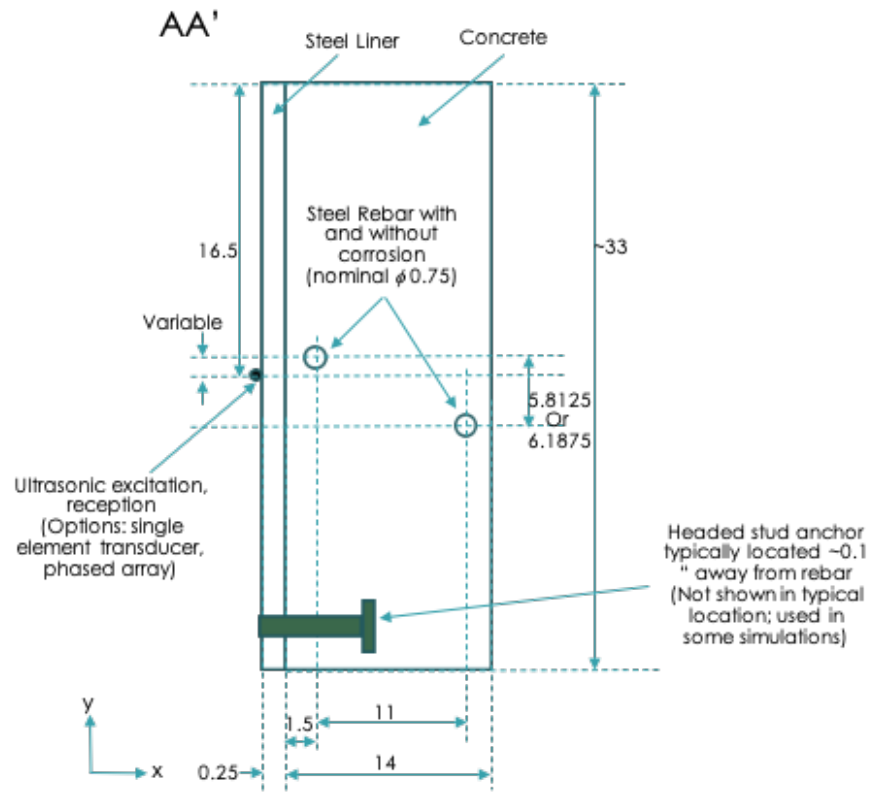

Figure 2. 2D slice along the $\mathrm{AA}^{\prime}$ plane used in the simulations.

\subsubsection{Transducer and Sensor Setup}

The transducer used for ultrasonic inspection is assumed to be an ultrasonic array transducer, positioned on the steel liner and interrogating the concrete through the liner. The system configuration selected resembles the characteristics of commercially available ultrasound systems for nondestructive evaluation of concrete structures. The transducer configuration is documented in table 2. The same transducer is used in pulse-echo mode for reception, which means that one transducer emits while the other transducers listen for echoes. We assumed symmetry. This reduces simulation to the recording with transducers $k, k+1, \ldots, N$ when the source is transducer $k$ and where $N$ is the total number of transducers.

\subsubsection{Material Properties}

The material properties used in the simulation are summarized in table 3 . While these values were used as the nominal material property settings in initial simulations, variability in the properties of concrete was handled through sampling from a normal distribution. This distribution of sound speed in concrete and concrete density assumed the quantities in table 3 as the mean value, with a standard deviation of 100-150 unit samples. The simulations assumed homogeneous materials, with nominal attenuation $(0.5 \mathrm{~dB} / \mathrm{cm})$ in the concrete and negligible attenuation in the steel liner and rebar. The variation in these parameters is expected to result in texture noise in the simulation results and provide the necessary variability for MBIR and ML algorithms. 


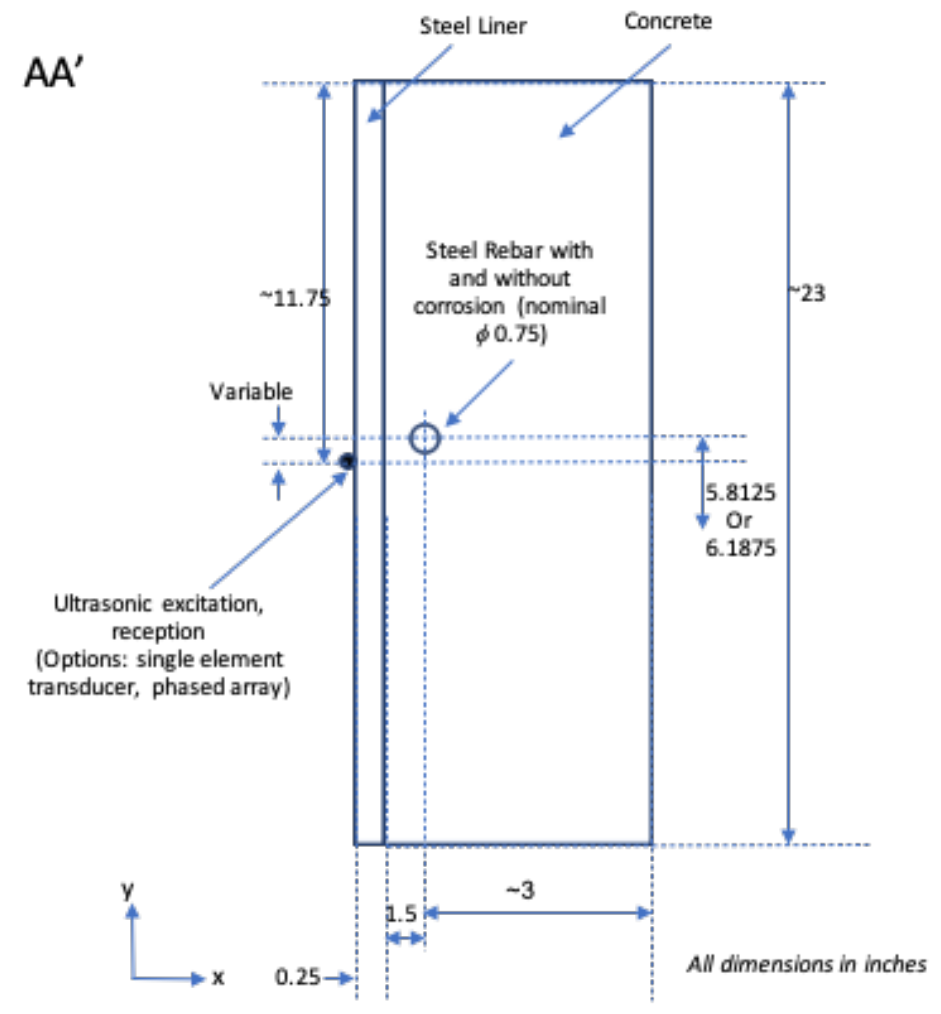

Figure 3. Truncated simulation geometry.

\subsubsection{Corrosion}

Corrosion in the rebars was assumed to be homogeneous and uniform around the circumference of the rebar. While this may not correspond completely with naturally occurring corrosion, the assumption of homogeneity is often used to calculate remaining life calculations. In this study, corrosion is assumed to initiate on the outer surface of the rebar and with time, assumed to grow. With the attendant assumption of homogeneity of the corrosion, the simulation uses a layered model to represent the corroded rebar. The thickness of the corrosion layer relative to the diameter of the rebar represents the fraction of the rebar that has corroded. Figure 4 and 5 show the ultrasonic phantoms with 50\% corrosion of the rebar. Figure 4 shows the full geometry that we discontinued due to lack of signal for the back rebar. Figure 5 shows the truncated geometry employed in this study. The corrosion layer is seen as a green ring around the uncorroded (yellow) core of the rebar. In these figures, which display the densities of the different materials, the steel liner is seen (yellow layer across the top of the figures) with a layer of air above that.

\subsubsection{Simulation Overview and Limitations}

Each simulation was performed using the k-Wave toolbox in MATLAB (Release 2018a) and executed on 16 nodes of a high-performance computing (HPC) system. It is worth noting that the k-Wave simulation software uses a spectral method for solving the wave propagation problem. The approach is not 


\begin{tabular}{|c|c|c|c|}
\hline Component & Dimensions & Units & Notes \\
\hline Thickness of steel liner & 0.25 & inches & \\
\hline $\begin{array}{l}\text { Thickness of concrete } \\
\text { slab (x-direction) }\end{array}$ & 4 & inches & \\
\hline $\begin{array}{l}\text { Vertical extent of con- } \\
\text { crete (y-axis) }\end{array}$ & 23 & inches & $\begin{array}{l}\text { Approximate. This dimension is adjusted } \\
\text { slightly to ensure that the number of grid } \\
\text { points is an integral power of } 2 \text {. }\end{array}$ \\
\hline $\begin{array}{l}\text { Grid spacing in } \mathrm{x}^{-} \\
\text {direction }\end{array}$ & 0.7668 & $\mathrm{~mm}$ & \\
\hline $\begin{array}{l}\text { Grid spacing in } y- \\
\text { direction }\end{array}$ & 0.813 & $\mathrm{~mm}$ & \\
\hline Rebar 1 depth & 1.5 & inches & $\begin{array}{l}\text { Liner surface to center of rebar; rebar is ori- } \\
\text { ented in the z-direction (out of the plane of } \\
\text { the page). }\end{array}$ \\
\hline Rebar 2 depth & 12.5 & inches & $\begin{array}{l}\text { Liner surface to center of rebar; rebar is ori- } \\
\text { ented in the z-direction. Rebar } 2 \text { was not in- } \\
\text { cluded in the reported simulations as it was } \\
\text { outside the truncated domain. }\end{array}$ \\
\hline Diameter of rebars & 0.75 & inches & Nominal; \#6 rebar \\
\hline $\begin{array}{l}\text { Spacing between rebars } \\
\text { in the y-direction }\end{array}$ & 5.8125 & inches & Center-to-center \\
\hline $\begin{array}{l}\text { Level of corrosion in re- } \\
\text { bars }\end{array}$ & $0,20,50,100$ & $\%$ & $\begin{array}{l}0 \% \text { corresponds to no corrosion. All nonzero } \\
\text { values refer to the amount of material that is } \\
\text { corroded from the outside of the rebar toward } \\
\text { the middle. }\end{array}$ \\
\hline Corrosion uniformity & Uniform & & $\begin{array}{l}\text { Rebar corroded uniformly all around the re- } \\
\text { bar, from the outside in (i.e., disc shape). }\end{array}$ \\
\hline $\begin{array}{l}\text { Delamination depth }(\mathrm{x}- \\
\text { direction) }\end{array}$ & 2 & $\mathrm{~mm}$ & $\begin{array}{l}\text { Arbitrarily selected; size of delamination be- } \\
\text { low the steel liner. }\end{array}$ \\
\hline $\begin{array}{l}\text { Delamination extent }(y- \\
\text { direction) }\end{array}$ & 40 & $\mathrm{~mm}$ & $\begin{array}{l}\text { Arbitrarily selected; size of delamination be- } \\
\text { low the steel liner. }\end{array}$ \\
\hline PML size & 20 & Grid Points & $\begin{array}{l}\text { Matched layer around the geometry, used to } \\
\text { eliminate ultrasonic reflections from the edge } \\
\text { of the simulation domain. }\end{array}$ \\
\hline
\end{tabular}

Table 1. Summary of simulation geometry parameters.

recommended for scenarios where the material properties change abruptly (i.e., step change). To address this concern, the simulations in this study assumed a gradual change (i.e., ramp change, with the intermediate simulation grid point corresponding to the average of quantities across the boundary) in the material properties across all boundaries (i.e., steel liner to concrete, concrete to rebar). The simulation results saved include the simulation settings, the complete response at each sensor grid point, and a processed version of the sensor readings that averages the responses from all grid points corresponding to a single transducer element. 


\begin{tabular}{|c|c|c|c|}
\hline Component & Value & Units & Notes \\
\hline Number of elements $(N)$ & 5 & & $\begin{array}{l}\text { Same sensor elements used for pulsing and re- } \\
\text { ceiving. }\end{array}$ \\
\hline Size of each element & 12.5 & $\mathrm{~mm}$ & $0.5 \%$ \\
\hline Distance between elements & 6.25 & $\mathrm{~mm}$ & $\begin{array}{l}0.25 \text { ". This is the edge-to-edge spacing, AKA } \\
\text { kerf width. }\end{array}$ \\
\hline Element pitch & 18.75 & $\mathrm{~mm}$ & Center-to-center spacing/pitch \\
\hline Array center & 15.75 & inches & $\begin{array}{l}\text { Centered in the simulation domain, in the } y- \\
\text { direction. In the } x \text {-direction, array is in contact } \\
\text { with the outside of the steel liner. }\end{array}$ \\
\hline Excitation frequency & 50 & $\mathrm{kHz}$ & Center frequency \\
\hline Wave mode & $\mathrm{L}, \mathrm{S}$ & & $\begin{array}{l}\text { Longitudinal and shear waves were both used } \\
\text { separately. Mode conversions accounted for in } \\
\text { simulation. }\end{array}$ \\
\hline Type of excitation & Tone burst & & 3-cycle tone burst \\
\hline Sampling frequency & 1 & $\mathrm{MHz}$ & $\begin{array}{l}\text { Sample frequency for simulation, sets the points } \\
\text { per wavelength and time-stepping values. }\end{array}$ \\
\hline Unnormalized source strength & 1 & $\mathrm{MPa}$ & $\begin{array}{l}\text { Signal strength normalized by the acoustic } \\
\text { impedance of the liner. }\end{array}$ \\
\hline Transducer operation & FMC & & $\begin{array}{l}\text { Full matrix capture: Each element is pulsed and } \\
\text { response recorded at all elements. }\end{array}$ \\
\hline
\end{tabular}

Table 2. Summary of transducer and inspection settings.

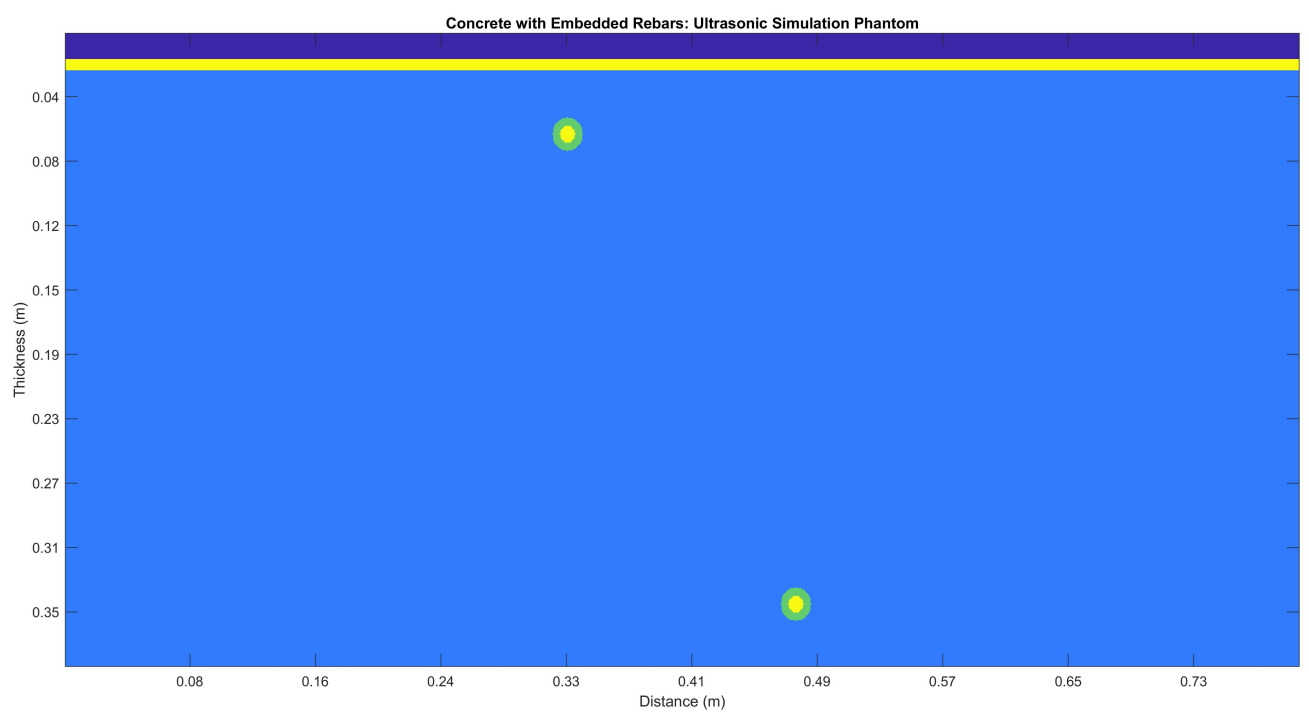

Figure 4. Original ultrasonic phantom of concrete slab with steel liner and rebars with corrosion (50\% corrosion). Recall this geometry is not used in this study. 


\begin{tabular}{|l|l|l|l|l|l|l|}
\hline Component & $\begin{array}{l}\text { Sound } \\
\text { speed } \\
(\mathrm{L}, \mathrm{m} / \mathrm{s})\end{array}$ & $\begin{array}{l}\text { Sound } \\
\text { speed } \\
(\mathrm{T}, \mathrm{m} / \mathrm{s})\end{array}$ & $\begin{array}{l}\text { Density } \\
(\mathrm{kg} / \mathrm{m3})\end{array}$ & $\begin{array}{l}\text { Young's } \\
\text { modulus } \\
(\mathrm{MPa})\end{array}$ & Reference & Notes \\
\hline \hline Concrete & & 2700 & 2000 & 50000 & $\begin{array}{l}\text { https://www.nd } \\
\text { t.net/article/ndt } \\
\text { ce03/papers/v0 } \\
65 / \mathrm{v065.htm}\end{array}$ & $\begin{array}{l}\text { Shear speed distributions as- } \\
\text { sumed a standard deviation of } \\
100 .\end{array}$ \\
\hline Steel liner & 5700 & 3200 & 7870 & 200000 & $\begin{array}{l}\text { Standard } \\
\text { charts of mate- } \\
\text { rial properties }\end{array}$ & $\begin{array}{l}\text { Assuming nominal values for } \\
\text { steel. }\end{array}$ \\
\hline $\begin{array}{l}\text { Uncorroded } \\
\text { rebar }\end{array}$ & 5700 & 3200 & 7870 & 200000 & $\begin{array}{l}\text { Standard } \\
\text { charts of mate- } \\
\text { rial properties }\end{array}$ & $\begin{array}{l}\text { Assuming steel liner and rebar } \\
\text { are same material. }\end{array}$ \\
\hline $\begin{array}{l}\text { Corroded } \\
\text { rebar }\end{array}$ & 5700 & 3200 & 5000 & 200000 & $\begin{array}{l}\text { François et al. } \\
\text { (2018b) }\end{array}$ & $\begin{array}{l}\text { Study based on 27-year-old } \\
\text { corroded rebars. Other studies } \\
\text { seem to indicate corrosion it- } \\
\text { self has Young's modulus be- } \\
\text { tween 1-9 GPa. }\end{array}$ \\
\hline
\end{tabular}

Table 3. Material properties used in k-Wave simulations.

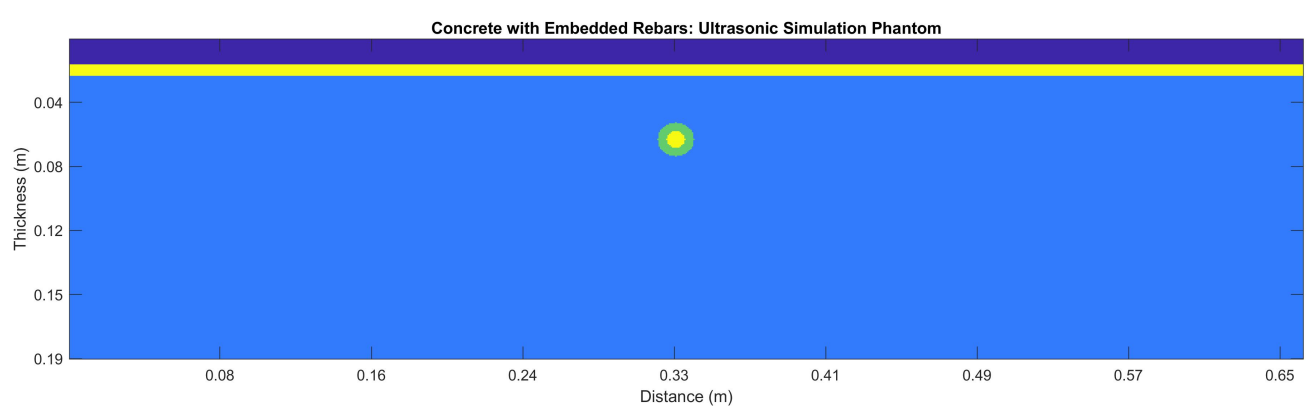

Figure 5. Ultrasonic phantom of truncated simulation domain, showing the steel liner and corroded rebar. Final phantom for study.

- While the simulation setup is relatively flexible and allows for the simulation of a variety of conditions, there are inherent limitations associated with finite element-based models that can limit the accuracy of the solution. In the case of k-Wave, the spectral method used limits the ability to simulate large gradients in density or speed. Such conditions might occur, for instance, when simulating boundaries between high- and low-density materials (e.g., an air pocket in concrete). While the issue may be mitigated through the use of gradual transitions (as done in this study), this inherently limits the accuracy of the simulation. Additional sources of uncertainty in the computed responses arise from:

- Limitations in modeling the transducer response and bandwidth. The simulations assume a set of point sources and sensors and that the applied excitation is a stress wave excitation. In reality, a 
transducer with a finite bandwidth, and conversion efficiency converts the applied signal to a stress wave excitation and vice versa.

- Potential imperfect application of boundary conditions. While PMLs were used in the simulations, no systematic study of the optimal PML thickness was performed. As a result, the computed responses may have residual contributions from reflections from the domain boundaries. In addition, interfaces (e.g., steel liner to concrete) are assumed to be perfect; variability in contact angle or localized disbonding may decrease sensitivity to the quantity of interest (i.e., corrosion in rebar).

- Inhomogeneity and anisotropy in material properties. This study assumes homogeneous conditions. While this is a reasonable assumption for steel, it is reasonable, in the case of concrete, to expect pockets of inhomogeneity, and the validity of this assumption for corrosion is unknown. However, the simulations may still be considered a good first approximation given the large wavelength of low-frequency ultrasound in concrete and the small size of inclusions relative to the wavelength. The expected randomness in size and distribution of any inclusions through the volume also likely results in conditions that are largely seen as homogeneous by the propagating wave. Similarly, inhomogeneity in the corrosion layer may also impact the response, and the extent of impact will need to be quantified in future studies. The study also assumes the material properties are isotropic (i.e., independent of wave propagation direction). Again, while a reasonable first approximation, concrete and possibly corrosion products exhibit anisotropy in ultrasonic wave propagation. Future studies in this area should explicitly account for inhomogeneity and anisotropy to better characterize the sensitivity of the methods described in this document to corrosion in rebars.

- Material property uncertainties can add further uncertainty to the computed result. For example, this study assumed a baseline set of values for concrete density and sound speed with some variability around these values. In practice, the concrete may be saturated with water, or the specific concrete mix may be inconsistent with the values used in this study. Future studies will need to account for these variabilities as well.

- Environmental conditions (temperature) can change the ultrasonic signal-to-noise ratio; the extent of this change will need to be quantified.

Other potential improvements in the measurement method that may be targets for future study include increasing the number of transducer elements; modifying the excitation signal (e.g., chirp excitations may provide additional insights); and explicitly monitoring the harmonic content for changes due to corrosion, either directly or through nonlinear mixing in the structure.

\section{SYNTHETIC EXPERIMENTS}

The performance of MBIR was tested with synthetic data generated by the K-wave simulator. Figure 6 demonstrates the object structure that was used in the simulation. The specimen width is $60 \mathrm{~cm}$, the depth is $10.8 \mathrm{~cm}$, and it has concrete and steel materials. The concrete has an acoustic speed of $2700 \mathrm{~m} / \mathrm{s}$, a density of $2000 \frac{\mathrm{kg}}{\mathrm{m}^{3}}$, and an attenuation coefficient of $0.5 \frac{\mathrm{dB}}{\mathrm{cm}}$. The acoustic speed in steel is $3200 \mathrm{~m} / \mathrm{s}$, and its density is $7870 \frac{\mathrm{kg}}{\mathrm{m}^{3}}$ with $0 \frac{\mathrm{dB}}{\mathrm{cm}}$ attenuation coefficient. In Figure 6, the first layer from the top is a steel liner (in black) with a thickness of $0.63 \mathrm{~cm}$. Following the steel, there is a concrete layer (in gray) with a thickness of $10.16 \mathrm{~cm}$. The concrete was embedded with a steel rebar (in black), which has some corrosion 
(in orange). The rebar diameter is $1.9 \mathrm{~cm}$, and it is aligned with the center of the specimen. We produced four different outputs from $\mathrm{k}$-Wave, where each output corresponds to a different corrosion percent: $0 \%, 20 \%, 50 \%$, and $100 \%$. At the top center of the specimen, five equally spaced transducers (separated by $0.625 \mathrm{~cm}$ ) were placed to transmit and receive. That means there were 20 received signals, whereas we only considered distinctive pairs (i.e., 10 pairs). The ultrasound system is considered to comprise nondirectional isotropic transmitters and receivers. The input signal to the system is a three-cycle sine wave with central frequency of $50 \mathrm{KHz}$ and sampling rate of $1 \mathrm{MHz}$. The output timeseries signals generated by k-wave had a $\Delta t=15.62 \mathrm{~ns}$, which equates to $64 \mathrm{MHz}$ signals. To make the synthetic data more realistic, we added uncertainty (i.e., texture) to the concrete acoustic speed and density. In particular, we added white Gaussian noise with a zero mean and a standard deviation of 100 units-resulting in a $\pm 3.7 \%$ and $\pm 5 \%$ variance for speed and density, respectively. For each corrosion level, we generated 57 random simulations, for a total of 228 simulations.

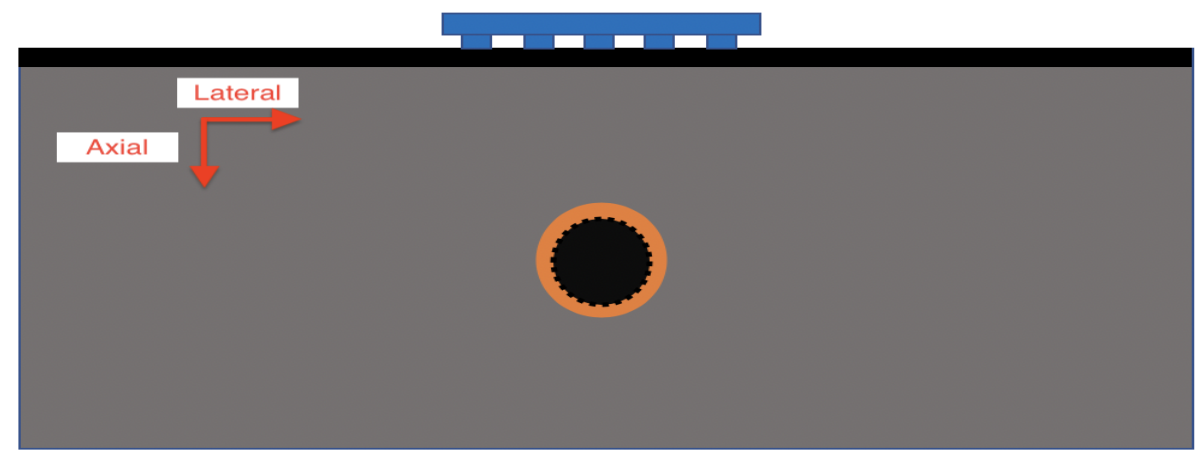

Figure 6. Structure of the specimen.

\subsection{MBIR RECONSTRUCTIONS}

In this section, we briefly discuss the mathematical components of MBIR. For an in-depth discussion of the MBIR algorithm and its mathematical formulation, please refer to our previous technical publications in Almansouri et al. (2018b,a,c).

The maximum a posteriori (MAP) estimate employed in our MBIR algorithm is given by:

$$
\begin{aligned}
x_{\mathrm{MAP}} & =\underset{x}{\arg \min }\{f(x)\} \\
& =\underset{x}{\arg \min }\{-\log p(y \mid x)-\log p(x)\},
\end{aligned}
$$

where $f(x)$ is the MAP cost function, $y$ are the system measurements, $x$ is the object image to reconstruct, $p(y \mid x)$ is the probability density function (pdf) or forward model of the measurements given an image estimate of the object, and $p(x)$ is the pdf likelihood or prior model that an image pixel exists inside a neighborhood of pixels.

Given that our forward model is assumed linear, the measurements $y$ can be obtained by the following equation:

$$
y=A x+D g+w,
$$


where $A$ is the system matrix, $D$ is the transducer crosstalk or surface wave model, $g$ is the scaling coefficient vector for $\mathrm{D}$, and $w$ is an i.i.d Gaussian vector with distribution $w \sim \mathcal{N}\left(0, \sigma^{2} I\right)$. Hence,

$$
-\log p(y \mid x)=\frac{1}{2 \sigma^{2}}\|y-A x-D g\|^{2}+\text { constant. }
$$

As shown in Figure 7, $A$ is computed by considering a signal transmitted from transducer $i$ at location $r_{i} \in \mathbb{R}^{3}$, reflected by point $v \in \mathbb{R}^{3}$, and received from transducer $j$ located at $r_{j} \in \mathbb{R}^{3}$.

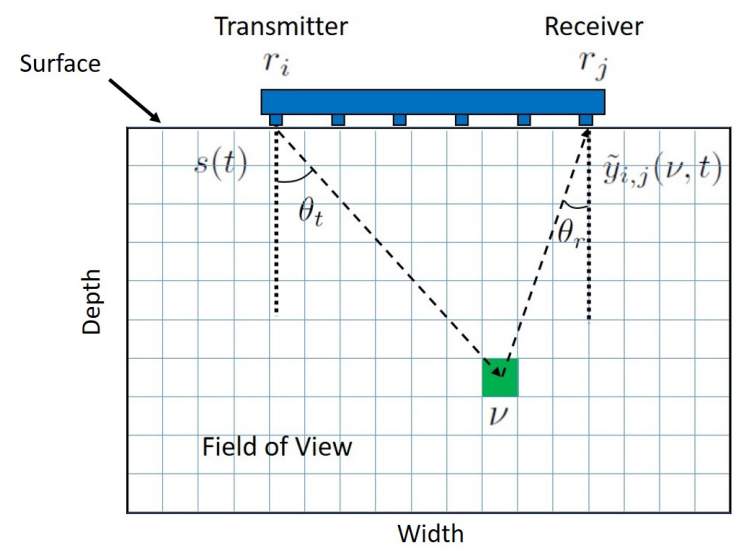

Figure 7. An illustration of a typical ultrasonic one-sided flight path diagram. $s(t)$ is the transmitted signal, $v$ is a point in the field of view, $y_{i, j}(v, t)$ is the received signal reflected from $v, \theta_{t}$ is the angle between $r_{i}$ and $v$, and $\theta_{r}$ is the angle between $r_{j}$ and $v$.

Next, we adopted the q-generalized Gaussian Markov random field (QGGMRF) for the prior model from Thibault et al. (2007). With this design, the prior model is

$$
p(x)=\frac{1}{z} \exp \left(-\sum_{\{s, r\} \in C} b_{s, r} \rho\left(x_{s}-x_{r}\right)\right),
$$

where $z$ is a normalizing constant, $C$ is the set of pair-wise cliques, and

$$
\rho(\Delta)=\frac{|\Delta|^{p}}{p \sigma_{g_{s, r}}^{p}}\left(\frac{\left|\frac{\Delta}{T \sigma_{g s, r}}\right|^{q-p}}{1+\left|\frac{\Delta}{T \sigma_{g_{s, r}}}\right|^{q-p}}\right),
$$

where

$$
\begin{aligned}
\sigma_{g_{s, r}} & =\sigma_{0} \sqrt{m_{s} m_{r}} \\
m_{s} & =1+(m-1) *\left(\frac{\text { depth of pixel s }}{\text { maximum depth }}\right)^{a}
\end{aligned}
$$

Hence,

$$
-\log p(x)=\sum_{\{s, r\} \in C} b_{s, r} \rho\left(x_{s}-x_{r}\right)+\text { constant. }
$$


Substituting the forward and prior models into Eq. 2,

$$
\begin{aligned}
(x, g)_{M A P} & =\underset{x, g}{\arg \min }\left\{\frac{1}{2 \sigma^{2}}\|y-A x-D g\|^{2}\right. \\
& \left.+\sum_{\{s, r\} \in C} b_{s, r} \rho\left(x_{s}-x_{r}\right)\right\} .
\end{aligned}
$$

We employed the majorization technique and the iterative coordinate descent (ICD) algorithm to solve Eq. 8.

\subsubsection{MBIR Configuration}

In an experiment with a real system and specimen, the specimen resides in a spatially continuous space (i.e., no discrete grid points). Although a spatially continuous specimen cannot be simulated in k-Wave, a specimen with grid space several orders of magnitude smaller than the reconstruction technique grid space (i.e., reconstructed image pixel size) can mimic the spatial relationship between a real and synthetic specimen. For our experiments, the grid space in k-Wave was set to approximately $0.5 \mathrm{~mm}$ in the axial direction and $0.6 \mathrm{~mm}$ in the lateral direction (See Figure 6). While, in MBIR, the reconstruction resolution is fixed at $3 \mathrm{~mm}$ or $10 \mathrm{~mm}$ in both directions. The nominal resolution expected for our application is approximately $30 \mathrm{~mm} @ 50 \mathrm{kHz}$. From previous experience, it is a good practice to select a pixel size three times smaller than the nominal resolution. Figure 8 illustrates the impact of pixel size on the reconstruction quality. This coarser resolution also speed up reconstruction speed by reducing the computational complexity of the model as it is the case for real experiments. Furthermore, we reduce the field of view in MBIR to approximately $30 \mathrm{~cm}$ in the axial direction and $12 \mathrm{~cm}$ in the lateral direction or depth. We also set the pixel center to be the top left corner of each pixel. For in-depth technical details on our MBIR algorithm, please, refer to Almansouri et al. (2018a).

Ground Truth

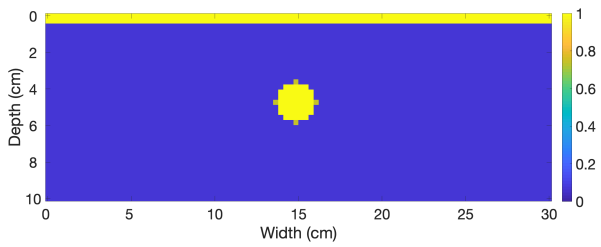

(a)

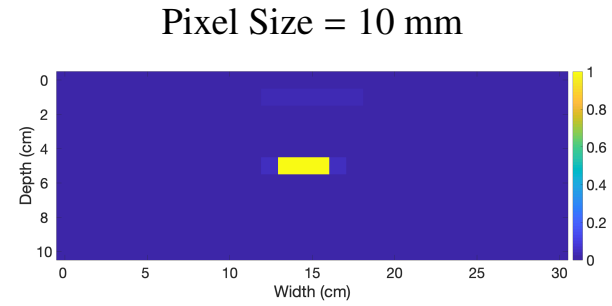

(c)

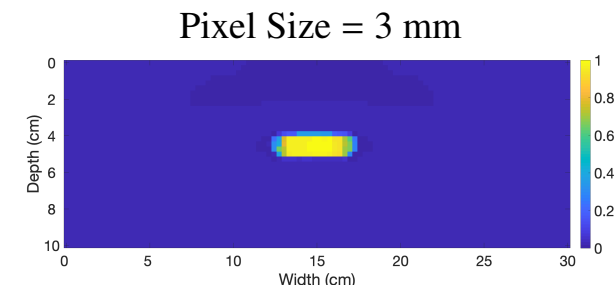

(b)

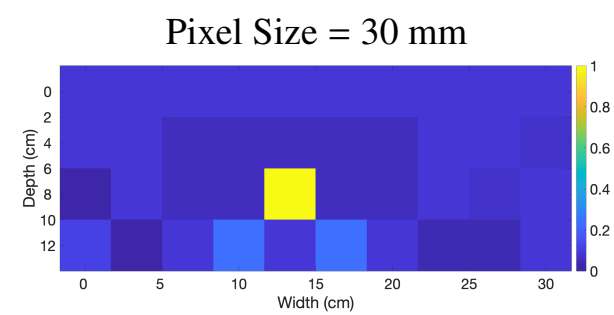

(d)

Figure 8. Examples of image reconstructions for different MBIR image pixel sizes. 


\subsubsection{MBIR Regularization parameters}

MBIR is a powerful algorithm. However, the configuration of the regularization model is critical for a successful reconstruction of the specimen. These parameters need to be adjusted after any significant changes to the imaging pipeline, such as imaging with a new system or at a different central frequency and bandwidth, and after imaging of a new material (e.g., imaging concrete alone vs. imaging concrete behind a steel liner). In Almansouri et al. (2018a), we document the parameters for our regularization technique. Table 4 demonstrates the prior model parameters we used in this experiment. Note that $\sigma$ is the variance of $w$ in Eq. 8, and it has to be between the maximum and minimum value in $y$ (selected based on the noise level in received signals). To ensure convexity and continuity of first and second derivatives of the prior model, the condition $1 \leq p \leq q=2$ must be satisfied. The parameter T controls the edge threshold. Also note that $\sigma_{0}, m$, and $a$ are the QGGMRF parameters, where $m$ is the variance of the image for deeper regions. To this end, these parameters can be adjusted to emphasize certain sections of the specimen. For example, the steel liner produces a strong reflection and several reverberations; consequently, contributing with strong reconstruction artifacts (see Figure 9(a)). These artifacts can be suppressed at the cost of losing the steel liner interface in the reconstruction (see Figure 9(b)). For the results shown below, the regularization parameters were set to emphasize the rebar region and suppress artifacts without compromising the visibility of the steel liner.

Table 4. MBIR parameter settings used in K-wave data reconstructions.

\begin{tabular}{|c|c|c|}
\hline Parameter & MBIR & Unit \\
\hline Number of iterations & 100 & - \\
\hline$\sigma$ & $4 \times 10^{-6}$ & Pascal \\
\hline $\mathrm{p}$ & 1.1 & - \\
\hline $\mathrm{q}$ & 2.0 & - \\
\hline $\mathrm{T}$ & $1 \times 10^{-4}$ & - \\
\hline$\sigma_{0}$ & $2 \times 10^{-4}$ & $m^{-3}$ \\
\hline$m$ & 0.01 & - \\
\hline$a$ & 3 & - \\
\hline
\end{tabular}

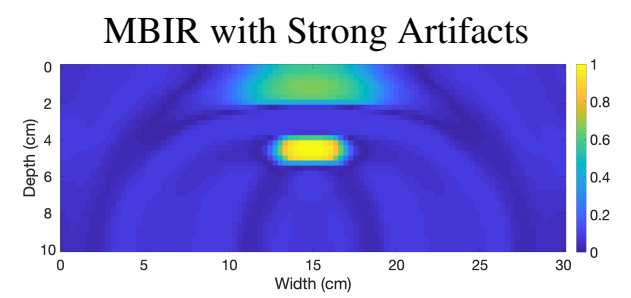

(a)

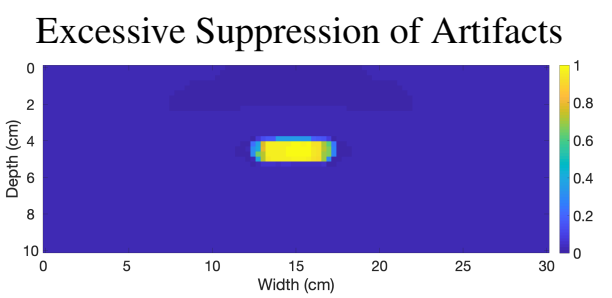

(b)

Figure 9. Examples of image reconstructions for extreme MBIR regularization parameters. 


\subsubsection{Average Intensity Measure}

Many quantitative assessment methods have been proposed in the literature. However, we developed a metric that better suited this study-the Average Intensity Measure (AIM). In particular, the AIM quantitatively assess a proper reconstruction of the rebar as well as its corrosion percent. Given that ultrasound is a three-dimensional problem and our reconstructions are planes, we are solving an ill-posed problem. Consequently, the solution for the Intensity Reflectively Coefficients (IRC) cannot be expected to match the expected range from zero to one. However, the reconstructed IRC values should correlate with the actual acoustic values and relative differences between IRCs for different material interfaces should remain. Therefore, we scaled the reconstructed images relative to the ground truth image we are comparing the reconstruction to. Note that the cement-steel and cement-corrosion IRCs are 0.6469 and 0.4953 , respectively. We compute the AIM score with the following steps:

1. We first set a region of interest (ROI) centered at and covering the rebar.

2. We segment the image between cement, corroded steel, and steel.

3. Next, let $S_{G T}$ be the total number of steel pixels in ground truth (i.e., number of pixels that are equal to $I R C_{\text {steel }}=0.6469$ ), and $C_{G T}$ be the total number of corroded pixels in ground truth (i.e., number of pixels that are equal to $\left.I R C_{\text {corrosion }}=0.4953\right)$.

4. Then, we compute $a=\frac{C_{G T}}{S_{G T}+C_{G T}}$ as the average number of corroded pixels in ground truth.

5. The next step is to scale the reconstructed image $X_{\text {scaled }}=X * \frac{\max (G T)}{\max (X)}$

6. Similarly, let $S_{X}$ be the total number of steel pixels in a reconstructed image (i.e., number of pixels with value $p$ where $I R C_{\text {steel }}-\epsilon<p<I R C_{\text {steel }}+\epsilon$, where $\epsilon$ is a small value (e.g., 0.1 ), and $C_{X}$ is the total number of corroded pixels in a reconstructed image (i.e., number of pixels that are between $\left.I R C_{\text {corrosion }}-\epsilon<p<I R C_{\text {corrosion }}+\epsilon\right)$.

7. Then we compute $b=\frac{C_{X}}{S_{X}+C_{X}}$ as the average number of corroded pixels in the reconstructed image.

8. Finally, $A I M=1-|a-b|$.

The rationale behind finding the pixels between $p-\epsilon$ and $p+\epsilon$ is that reconstructed pixels may differ from their original intensities with a certain tolerance due to model imperfections and rounding errors. Note that if $a=0$ (i.e., $0 \%$ corrosion), then $A I M$ should be close to 1 when the reconstructed image has almost no corrosion $(b=0)$. The inverse argument applies to the $100 \%$ corrosion case.

In section 4.1.2.1, we discuss the effect of $\epsilon$ value on the accuracy of our method.

\subsubsection{Application of AIM to Synthetic Experiments}

This section documents the steps we take to ensure the correctness of the MBIR wave propagation model and the AIM metric on synthetic data. The simulated data was generated by creating some ground truth image $\boldsymbol{g}$ and multiplying it by our system matrix $\boldsymbol{A}$ to generate the timeseries signals $\boldsymbol{y}$ (i.e., $\boldsymbol{y}=\boldsymbol{A} \boldsymbol{g}$, where $\boldsymbol{g}$ is the ground truth image stacked in a vector). The ground truth is assumed to be a homogeneous concrete medium (with no steel liner) with two phantoms placed in the center of the field of view. The size of each phantom is just one pixel (see Figure 10(a)). The phantom on the left has an intensity of 1, and the one on the right is 0.5. The red rectangle in Figure 10(a) represents the bounding box around our region of 
interest. Next, we perturb $\boldsymbol{y}$ with additive white Gaussian noise (AWGN) with a zero mean and a standard deviation of 0.01 ( $y$ is in the range [-1,1]). Figure 10(b) and Figure 10(c) depict our reconstructed images for noiseless and noisy data, respectively. Visually, it is clear that MBIR is capable of preserving the high and low intensities with insignificant artifacts. In terms of numbers, AIM $=99.17 \%$ for the reconstruction in Figure 10(b) and 97.60\% for the noisy data reconstruction in Figure 10(c), both with $\epsilon=0.1$. Note that our AIM approach accounts for the accuracy of reconstructions regardless of insignificant artifacts when $\epsilon$ is sufficiently high (e.g., 10\% of maximum possible intensity). However, one can opt to quantitatively assess reconstructions using AIM while taking into account the presence of noise by setting $\epsilon$ to a sufficiently small value (e.g., $1 \%$ of maximum possible intensity). Empirically, a reasonable value of $\epsilon$ for such application lies in the range $[0.01,0.1]$ when the pixels value range is $[0,1]$.

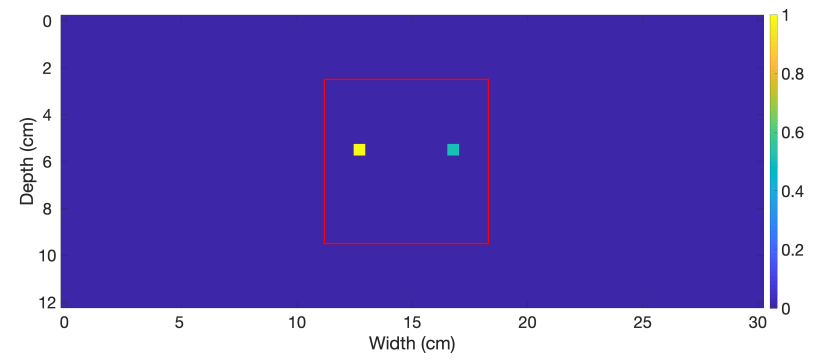

(a)

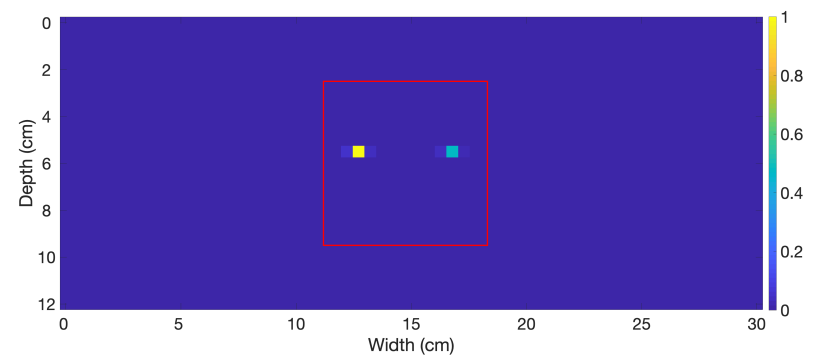

(b)

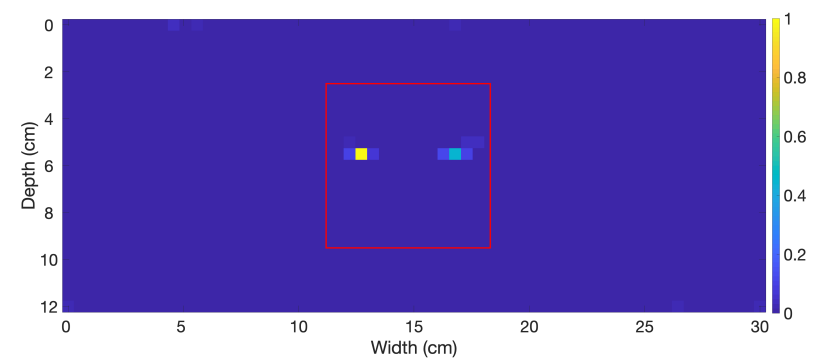

(c)

Figure 10. MBIR reconstructions of simulated data by a given (a) ground truth, where (b) is MBIR reconstruction of noiseless data $(\mathrm{AIM}=99.17 \%)$, and $(\mathrm{c})$ is MBIR reconstruction of noisy data (AIM $=97.60 \%$ ). 


\subsubsection{Reconstruction Results}

Figure 11 shows the $\mathrm{k}$-Wave simulations ground truth specimens (i.e., left column) and the corresponding MBIR reconstructions for 0\%, 20\%, 50\%, and 100\% corrosion levels (i.e., right column). See Section 1.1 for details on the simulation specimen and ultrasound system.

Ground Truth

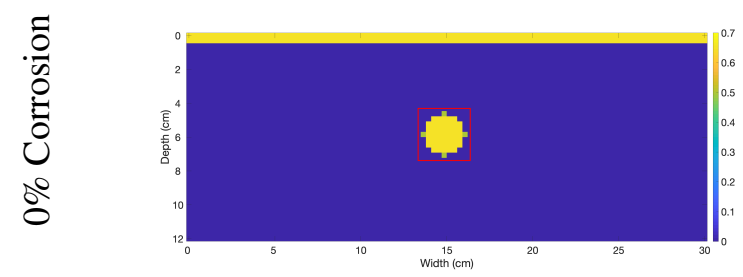

(a)

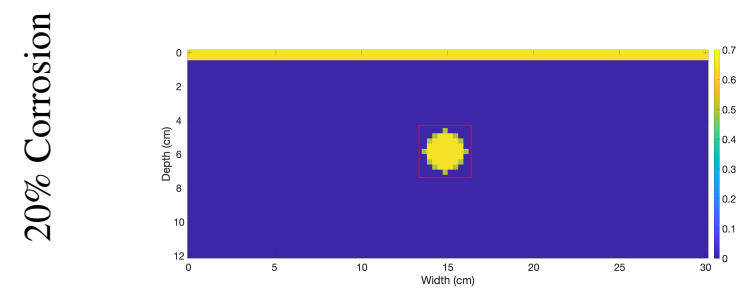

(b)

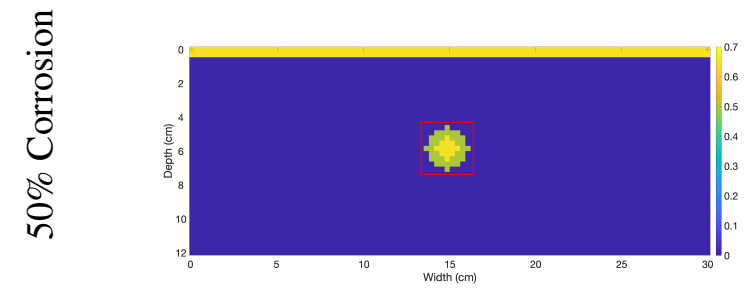

(c)

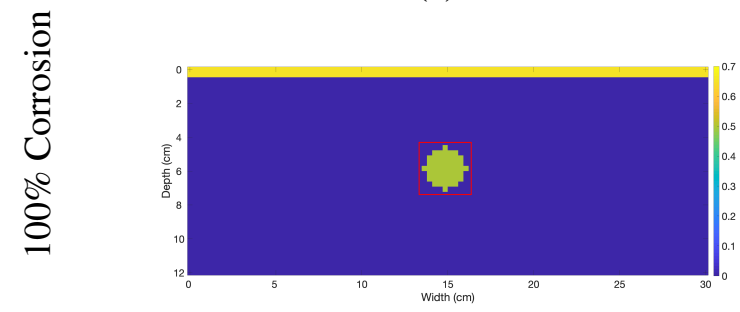

(d)
MBIR

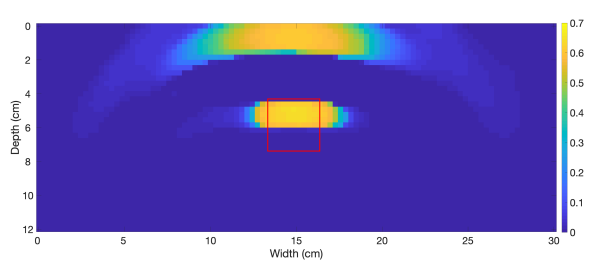

(e)

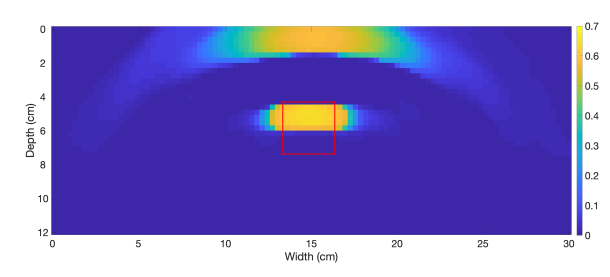

(f)

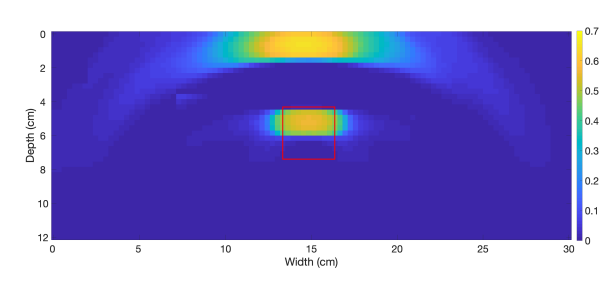

(g)

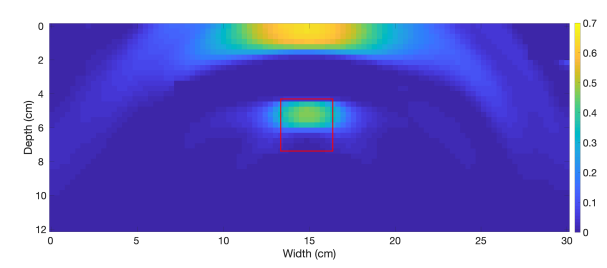

(h)

Figure 11. Ground truth and MBIR reconstruction images for different corrosion levels: Ground truth (a) 0\%, (b) 20\%, (c) 50\%, (d) 100\%; MBIR reconstructions (e) 0\%, (f) 20\%, (g) 50\%, (h) 100\%. 


\subsubsection{Qualitative Assessment}

A qualitative assessment of the MBIR reconstruction reveals that MBIR can reduce both texture noise and direct arrival signals artifacts and can resemble the location and size of rebar. Note that, because our forward model is assumed to be linear and homogeneous, MBIR may exhibit reflections from the steel liner as defects. However, after modifications to our forward model and prior model parameters, we can remove reflections from regions outside the area of interest. As a result, we can easily interpret our clean reconstructions and recognize the rebar. We also observe that the reconstructions closely match the corresponding corrosion-level ground truth images, even when the corroded disc thickness is orders of magnitude smaller than the nominal resolution for our system. The intensity of the pixels representing the acoustic IRCs of the specimen resemble the appearance of an interface with a closely matching acoustic impedance-as the corrosion level increases the intensity of the pixels decreases (i.e., from bright yellow to dark yellow or orange.).

\subsubsection{Quantitative Assessment}

The purpose of the AIM metric is to quantitatively assess the reconstructions. A summary of the quantitative assessment results is provided in Table 5. Note that the left AIM scores correspond at comparing ground truth images and reconstruction images that match in corrosion level, while the AIM scores at the right correspond to comparing all the reconstructions for all corrosion levels to the ground truth with a completely healthy rebar.

Table 5. Summary of AIM scores for images in Figure $11(\epsilon=0.1)$

\begin{tabular}{|rcrc|}
\hline Image Pair & Average AIM (\%) & Image Pair & Average AIM (\%) \\
\hline \hline (a) vs (e) & $\mu=86.18, \sigma=15.74$ & (a) vs (e) & $\mu=86.18, \sigma=15.74$ \\
(b) vs (f) & $\mu=85.54, \sigma=14.31$ & (a) vs (f) & $\mu=85.10, \sigma=14.03$ \\
(c) vs (g) & $\mu=84.92, \sigma=37.90$ & (a) vs (g) & $\mu=57.86, \sigma=16.79$ \\
(d) vs (h) & $\mu=88.46, \sigma=61.70$ & (a) vs (h) & $\mu=19.23, \sigma=6.17$ \\
\hline
\end{tabular}

The results in Table 5 show that MBIR consistently produce results that match the corresponding ground truth. It is important to note that the standard deviation of the left AIM scores increases with corrosion level. This behavior, although not ideal, it is expected given that the dynamic range of images with higher corrosion levels is smaller than images with the lower corrosion level. The added texture to the synthetic specimen will create greater changes in images with smaller dynamic range resulting in more spread AIM values. More interesting are the AIM scores at the right. These experiments resemble what will happen in an operational scenario. The expected intensity values are unknown and the best we can know is the presence or absence of a rebar. Therefore, if a rebar-like feature is observed in the reconstruction, we would like to know how the AIM score could be a predictor of corrosion level. As expected there is good separation between AIM scores for healthy rebar and unhealthy rebar (i.e., 50\% or more level of corrosion). However, there is substantial overlapping between the $0 \%$ and $20 \%$ AIM scores. These results are understandable given that the corrosion layer thickness is below our nominal resolution. This implies that we should explore other methods to predict corrosion level. With that said, it may be unrealistic to expect that corrosion will spread uniformly around the circumference of the rebar and that a side of the rebar may exhibit more damage than others. In that case a $20 \%$ corrosion response may be similar to the 
$50 \%$ corrosion response. This should be evaluated with empirical experiments.

Another quantitative assessment is to compute the average intensity and corresponding standard deviation of the rebar's neighborhood pixel intensities. Figure 12 summarizes the results, which show the expected behavior. As the corrosion level increases, the average intensity of the pixel decreases. This suggests that average pixel intensity around the rebar is another candidate feature for corrosion level estimation.

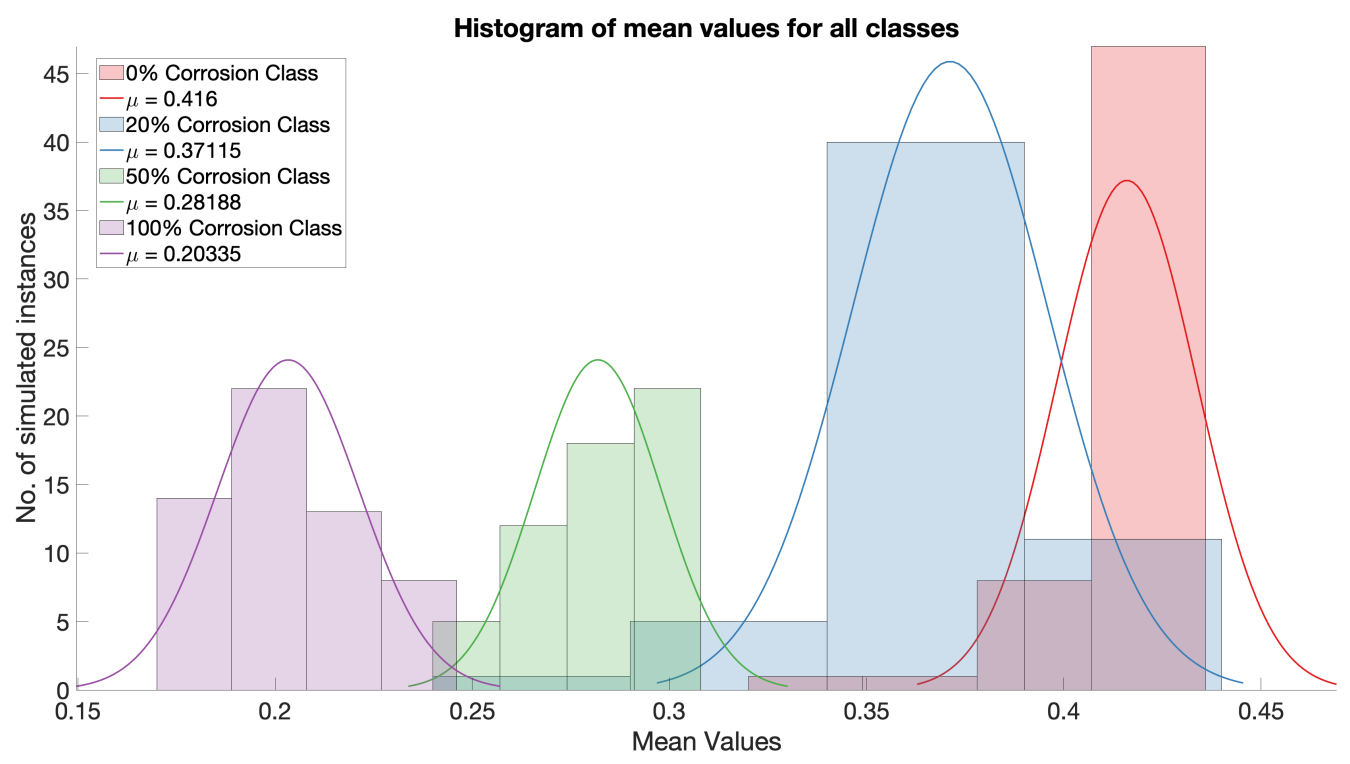

Figure 12. Reconstructed images average pixel intensity distributions per corrosion level.

\subsubsection{Reconstruction Results with Baseline Cancellation Method}

\subsubsection{Baseline Cancellation Method (BCM)}

In Subsection 4.1, the forward model is modified to cancel the direct arrival signals as shown in Equation 8. In principle, direct arrival signals are generated along with the transmitted wave. These direct arrival signals produce artifacts in the region close to the transducers. However, in this application, the transducers are attached to a steel liner which creates substantial artifacts around the sensors as shown in Figure 11. This is due to the fact that, beside the direct arrival signals, we have reflections form the edges of the steel liner that generate reverberations. Consequently, we believe that the forward model in 8 is not adequate to cancel all undesired signals. Hence, we propose a method to reduce the effect of these unwanted signals. The method assumes that we can characterize a baseline signal, which is a signal of the specimen without the feature of interest (e.g., the rebar). This is straightforward for simulated specimens. The baseline signal can be obtained by simulated the same specimen without the rebar. In order to account for the texture in concrete, we generate $n$ instances of the baseline signal. The final baseline signal could be the average signal of the $n$ set. However, this approach may be bias by the variations in concrete. Consequently, we employed a method that generate a baseline signal that contained the stronger features of the $n$ set. We adopt the well-know principal component analysis (PCA) method to find the stronger features (i.e., eigenvectors with higher eigenvalues). In particular, we select the eigenvectors that when combined 
produce the lowest mean square error (MSE) when compared to the original signal. Note that it can be shown that the MSE is the sum of the least eigenvalues. Then, the selected dominant eigenvectors are averaged to form a baseline signal. For our data set, we found that the largest 15 eigenvectors are enough to characterize the baseline signal. Finally, we construct the baseline matrix $\boldsymbol{B}$ to replace the direct arrival signal matrix $\boldsymbol{D}$. We will refer to this method as MBIR-BCM. Figure 13 illustrates the correction of the BCM method on one timeseries example. Note in Figure 14 that reflections proceeding from the the steel liner interface are heavily suppressed.
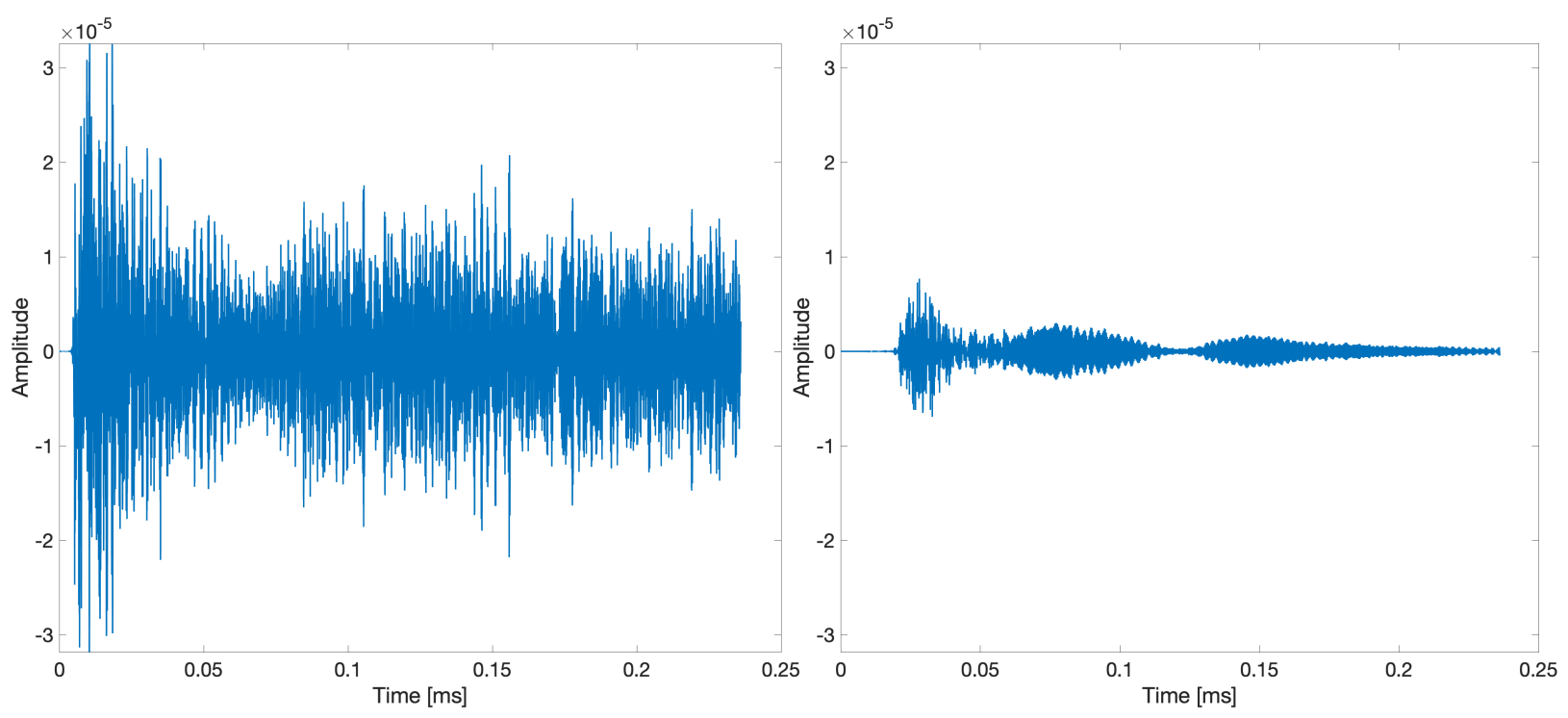

Figure 13. Baseline cancellation illustration. The left image is the original signal and the right image is the signal on the left after baseline cancellation.

In practice, this method is useful when the thickness and material of the steel liner are known. The ultrasound system interaction with the steel liner can be characterized and simulated to generate a baseline signal. This baseline signal can be integrated to the MBIR algorithm seamlessly as we did for the simulated experiments.

\subsubsection{Reconstructions with MBIR-BCM}

Figure 14 shows the k-Wave simulations ground truth specimens (i.e., left column) and the corresponding MBIR-BCM reconstructions for 0\%, 20\%, 50\%, and 100\% corrosion levels (i.e., right column).

\subsubsection{Qualitative and Quantitative Assessment}

The MBIR-BCM method offer slight visible improvements. The most significant difference is, as expected, the suppression of the reflections at the steel liner interface-completely removing the steel liner from the reconstruction. Moreover, the MBIR-BCM significantly improves the AIM scores, as shown in Table 6. In general, the AIM scores across different corrosion levels were more consistent with a tighter standard 
Ground Truth

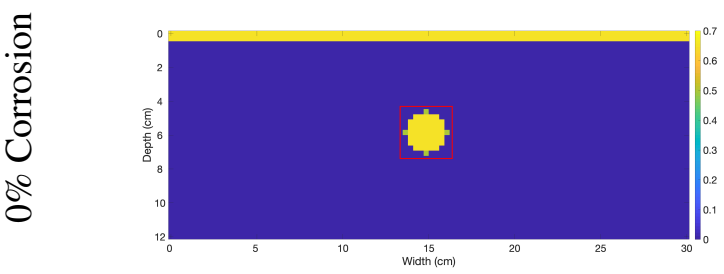

(a)

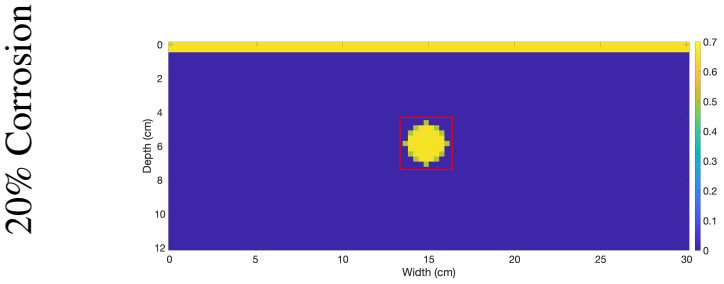

(b)

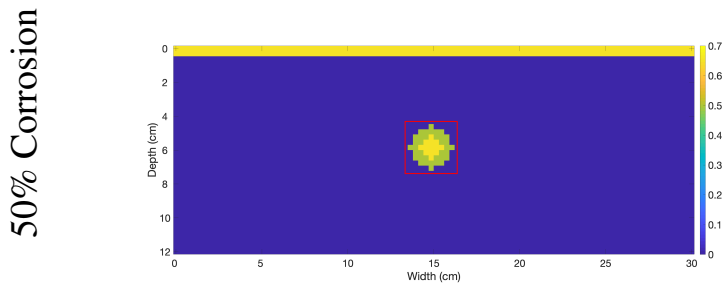

(c)

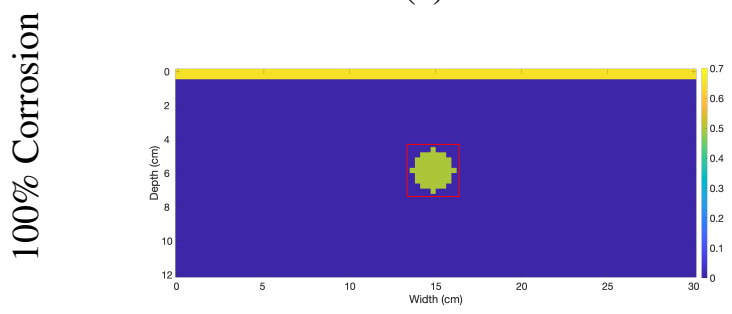

(d)
MBIR-BCM

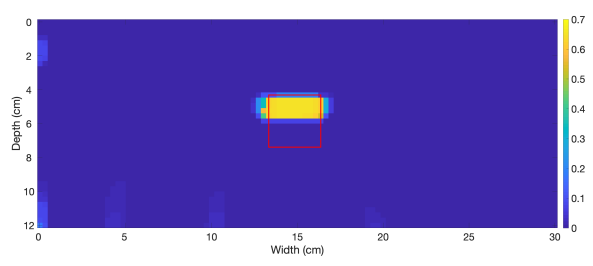

(e)

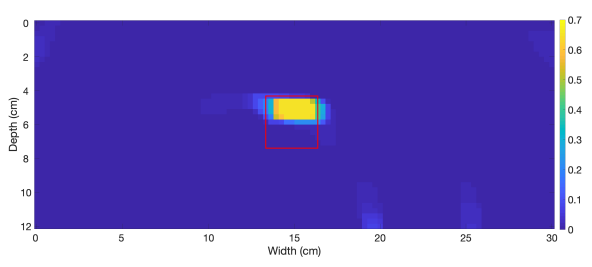

(f)

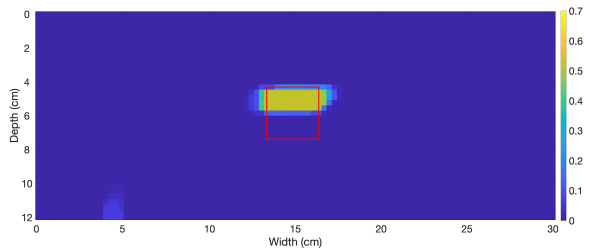

(g)

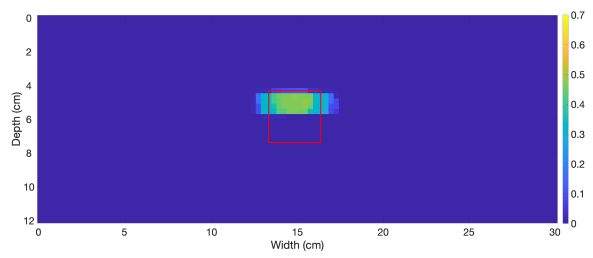

(h)

Figure 14. Ground truth and MBIR-BCM reconstruction images for different corrosion levels: Ground truth (a) $0 \%$, (b) 20\%, (c) 50\%, (d) 100\%; MBIR-BCM reconstructions (e) $0 \%$, (f) 20\%, (g) $50 \%$, (h) $100 \%$.

deviation and more separation between corrosion classes. On the other hand, as shown in Figure 15, the pixel intensity metric failed to better separate the corrosion classes. This may be due to our scaling method and should be explored in more detail as follow on work.

The MBIR-BCM method is not appropriate for applications where inspection of the steel liner is required. For those applications, the BCM method should follow a regular MBIR reconstruction after a feature of 
interest is identified. Note that data collection is done once and MBIR or MBIR-BCM methods can be applied off-line and as needed.

Table 6. Summary of AIM scores for images in Figure $14(\epsilon=0.1)$

\begin{tabular}{|rccc|}
\hline Image Pair & Average AIM (\%) & Image Pair & Average AIM (\%) \\
\hline \hline (a) vs (e) & $\mu=93.75, \sigma=4.89$ & (a) vs (e) & $\mu=93.75, \sigma=4.89$ \\
(b) vs (f) & $\mu=89.75, \sigma=11.01$ & (a) vs (f) & $\mu=89.15, \sigma=10.13$ \\
(c) vs (g) & $\mu=85.43, \sigma=14.99$ & (a) vs (g) & $\mu=48.96, \sigma=19.81$ \\
(d) vs (h) & $\mu=88.67, \sigma=12.20$ & (a) vs (h) & $\mu=19.02, \sigma=12.20$ \\
\hline
\end{tabular}

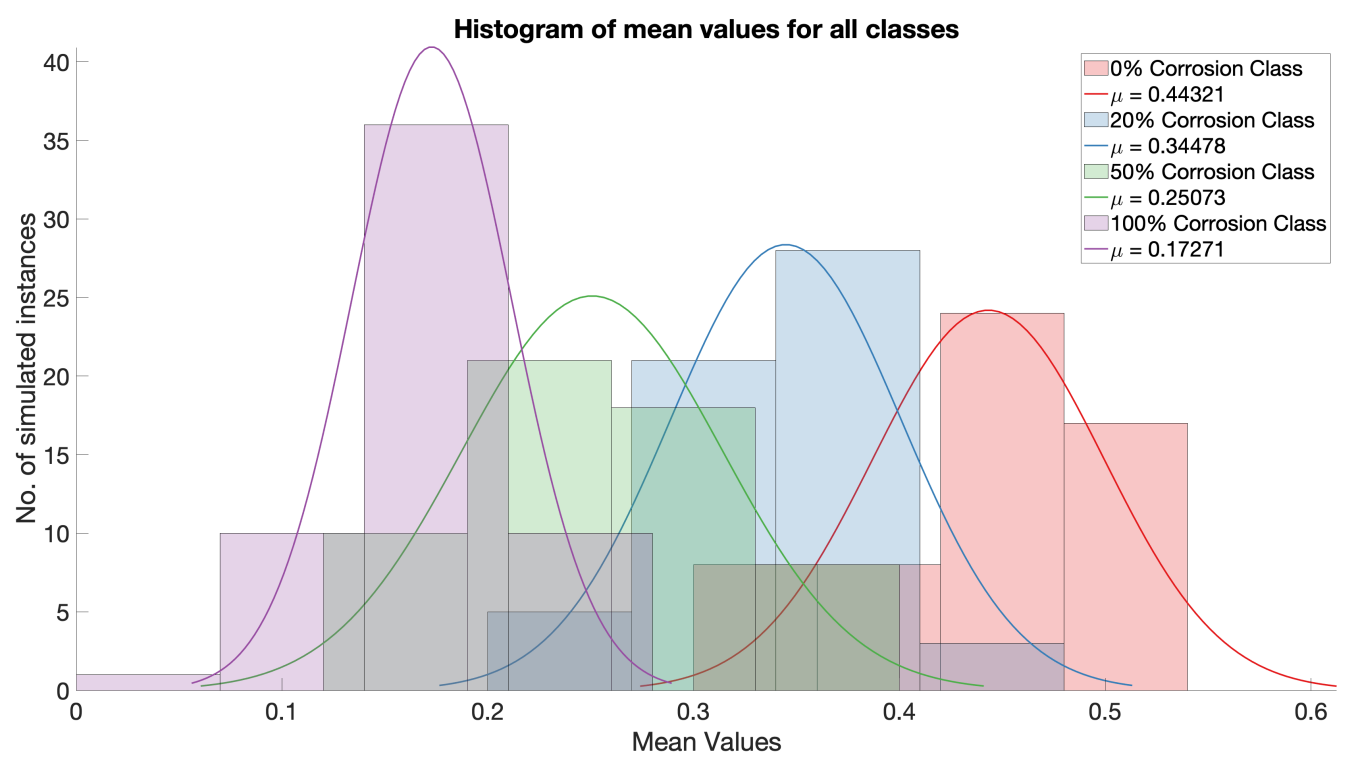

Figure 15. MBIR-BCM method reconstructed images average pixel intensity distributions per corrosion level.

\subsection{CORROSION CLASSIFICATION VIA MACHINE LEARNING}

ML and artificial intelligence (AI) algorithms are powerful tools for the classification of data into a set of known classes. For many applications, ML can handle tasks that will be impossible or too burdensome for a human user. This is especially the case for ultrasound time series signals. Humans can identify significant events in the data but may miss soft trends or trends that require connecting several pieces of non-evident data. While section 4.1 discusses the use of the MBIR technique for visual inspection of the rebar, this section documents the possibility of detecting the level of rebar corrosion directly from the time series data. Although we could design an ML/AI algorithm for classification of the corrosion level for the reconstructed image, we have shown in section 4.1 that a naive approach already provides separation between the corrosion levels. This may be true now, given that we are dealing with synthetic datasets. However, it is possible that for real data, we may need to train ML models to detect corrosion level from the MBIR images. Nevertheless, the time series signal will be too long for a direct classification. 
Therefore, MBIR could be used as a prepossessing step to identify features (e.g., rebar) of interest to narrow down the region of interest in the timeseries signals and then, perform analytics in a truncated version of the raw or filtered signal.

\subsubsection{Ultrasound Samples Extraction}

In contrast with the imaging section where we generated 57 instances for each corrosion level, for the machine learning study, we generated 100 instances for each of the 4 corrosion levels. For each instance, texture was introduced to the concrete region to mimic the expected velocity and density variances between different samples or sample locations. Recall that the system has 5 transducers for 10 unique transducer pairs, which result in the generation of 15 time series signals per data acquisition instance. This combination results in 6,000 unique time series-15,000 per corrosion level. Each time series has 15115 time samples for a time step $\Delta t$ of $15.62 E^{-9}$ seconds.

Although not impossible, classification of the whole time series signal is highly computationally expensive and it is unpractical for real applications where more than one feature of interest may be present. For the current synthetic and future real experiments, the first step is to extract a segment of the recorded time series that corresponds to echoes from waves interacting with the feature of interest (i.e., rebar) for each transducer pair $\{k, j\}$. Figure 16 illustrates the path of the ultrasound wave.

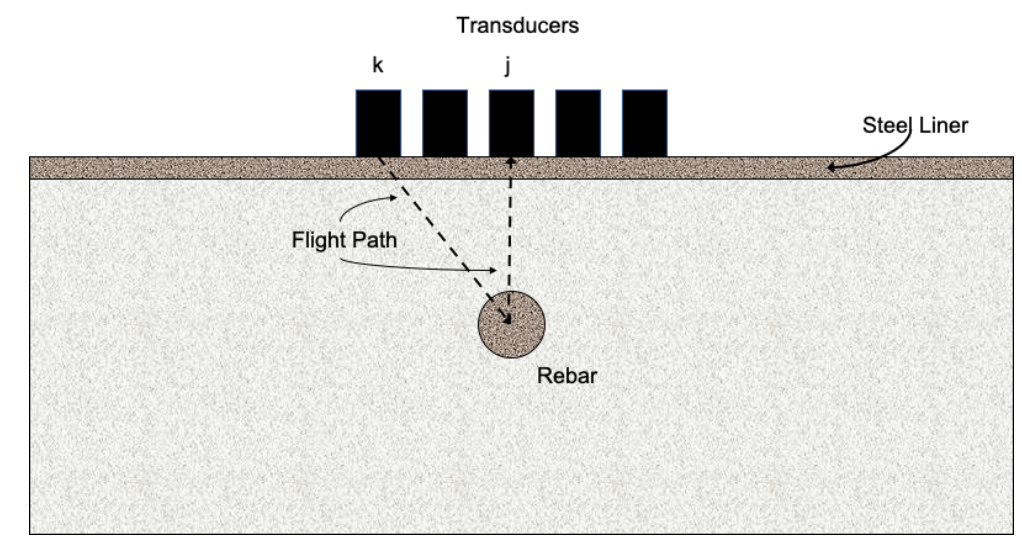

Figure 16. Illustration of wave flight path from source $k$, to rebar, to receiver $j$.

The steps to extract the event samples from the original signal are:

- Compute the inbound distance from emitting transducer $k$ to rebar center,

- Compute the outbound distance from rebar center to receiving transducer $j$,

- Compute, for the inbound and outbound paths, the segments occurring at the steel liner, concrete, and rebar,

- Compute the time spent at each material for inbound and outbound, and

- Sum all time delays to obtain $t_{R}$, which is the time index in the original time series signal where the recorded wave samples in the neighborhood of $t_{R} \in\left[t_{l b}, t_{r b}\right]$ may arrive from the rebar interface. 
Figure 17 illustrates how these steps affect the original signal. The blue wiggles show the original ultrasound signal sensed by transducer \#4, when transducer \#2 is emitting, while the purple overlay represents that segment of the original signal that will be extracted and further used for training the corrosion-level model. The left yellow, middle red, and right green vertical lines correspond to time indexes $t_{l b}, t_{R}$, and $t_{r b}$, respectively. Note that we follow these steps for all unique transducer pairs. We have two parameters, $\beta$ and $\alpha$, to adjust the position and length of the extraction window, respectively. The first parameter $\beta$ controls an offset from the rebar center $t_{R}^{\prime}=t_{R}^{\prime}-\beta * r_{R}$, where: $r_{R}$ is the radius of the rebar. The second parameter $\alpha$ controls the spacing between $t_{R}$ and $t_{l b}$ and $t_{r b}$, where

$$
\begin{aligned}
& t_{l b}=t_{R}^{\prime}-\alpha * \lambda, \\
& t_{r b}=t_{R}^{\prime}+\alpha * \lambda
\end{aligned}
$$

and $\lambda$ is the nominal wavelength of the ultrasound wave in concrete. We observed that our classification models performed better when the selection of $\beta$ and $\alpha$ result in a truncated signal composed of reflections originated at the edge of the rebar the length is at least $1.5 * \lambda$. For the results below we set up $\beta=2.5$ and $\alpha=0.5$. This resulted in a final truncated signal with a length of 1921 time samples.

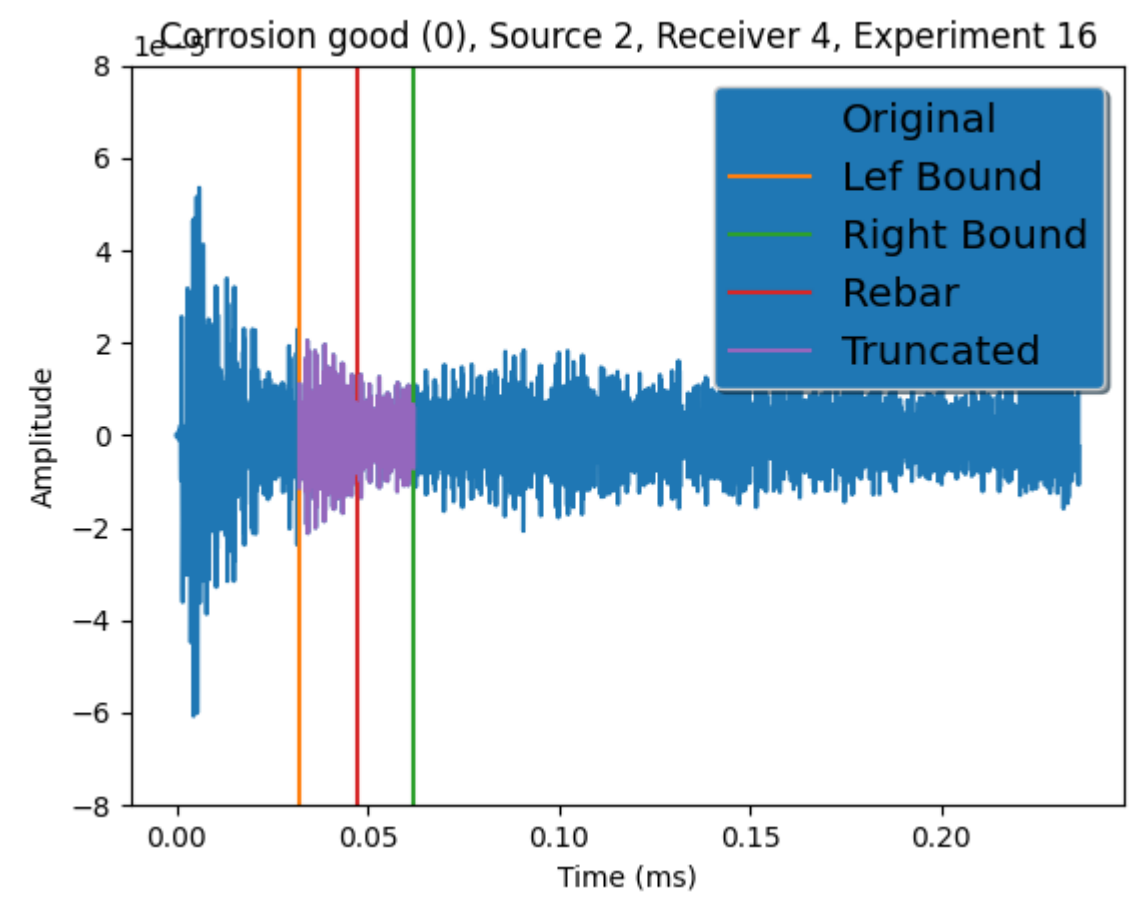

Figure 17. Illustration of data extraction from original signal: blue line is the original signal, the right and left vertical lines are the lower and upper bounds for the extraction window, the middle vertical line is the estimated time for the ultrasound wave to reach the rebar's center, and the purple line is the extracted signal. 


\subsubsection{Feature Engineering and Extraction and ML Model Training}

As shown in Figure 18, the machine learning model expects a feature vector that is composed of local data trends and global metrics from the raw truncated signal. Ideally, we want to identify features (e.g., frequency components, wavelet coefficients, average amplitude) that create the greater separation between the classes of interest (i.e., corrosion level). For this purpose, we employed the python TSFEL (Barandas et al. (2020)) library to automatically extract features from the time series signals. TSFEL extracts over 60 different temporal, statistical, and spectral features. After extraction of the features, TSFEL also provides built-in routines to remove correlated features, which reduces unnecessary computations for our training step. TSFEL found that for our application the most significant features are: Fast Fourier Transform (FFT) mean coefficients from 0 to 9, Empirical Cumulative Distribution Function (ECDF), ECDF Slope, ECDF percentile count 0 and 1, Centroid, Maximum Peaks, Mean Difference, Median Difference, Slope, and Entropy.

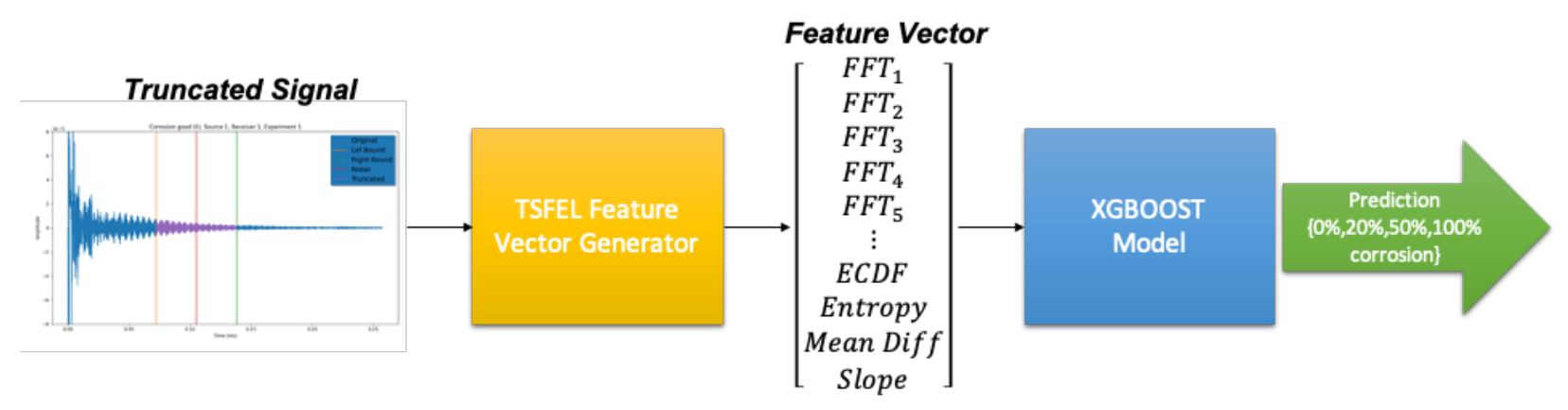

Figure 18. Illustration of machine learning prediction data flow.

After TSFEL feature vectors are generated, we input the vector to an XGBoostChen and Guestrin (2016) model to predict one of four corrosion levels. The model outputs $0,1,2$, and 3 , which correspond to $0 \%$, $20 \%, 50 \%$, and $100 \%$ level of corrosion, respectively. The model is trained on 4,062 (67.7\%) of the 6,000 individual timeseries; while 1,998 timeseries samples were left out for testing. Note that test samples are never used for training. In addition, the XGBoost algorithm was executed with its default configuration.

\subsubsection{Results}

The performance of the model is tested for three different scenarios. 1) Per-Signal Scenario, we assess the performance of the model for any individual timeseries, independent of the transducer pair, the location of the pair, and the predictions for other timeseries on the same scan instance. 2) The Per-Scan Scenario is an operational-like scenario, where we make a prediction taking in account all signals captured in an instrument scan. In our application, a scan contains signals for all 5 transducer-source/receiver combinations (i.e., total of 15 timeseries). Therefore, we combine the individual predictions by computing the average or median value of the scan prediction set-the average-fusion and median-fusion approaches. For the average-fusion method, $\mu\left(p_{k}\right)$ the predicted corrosion level for scan $k$ is given by

$$
\left.\mu_{(} p_{k}\right)=\left\lfloor\frac{1}{N} \sum_{i=1}^{N} p_{k, i}\right\rfloor,
$$


where $N$ is the number of unique transducers pairs (i.e., $N=15$ ), $\lfloor x\rceil$ is the nearest integer of $x$, and $p_{k, i}$ is the corrosion level prediction for timeseries $i$ in scan $k$. While for the median-fusion method, the predicted corrosion level $m\left(p_{k}\right)$ for scan $k$ is given by

$$
m\left(p_{k}\right)=\left\{\begin{array}{ll}
p_{k, \frac{N+1}{2}} & N \text { odd } \\
\frac{1}{2}\left(p_{k, \frac{N}{2}}+p_{k, \frac{N}{2}+1}\right) & N \text { even }
\end{array},\right.
$$

where the scan timeseries predictions are sorted in ascending order (i.e., $p_{k, i} \leq p_{k, i+1}$ and $i \in[1 \ldots N-1]$ ). Recall that for each corrosion level, we generate 100 scan instances (i.e., simulations). Therefore, for this scenario the testing 1,998 timeseries samples are reduced to 133 whole system testing samples. 3) The final scenario, is the Per-Pair Scenario, where we assess the performance of specific transducers pairs (i.e., source and receiver position and distance). For the performance results below, we generate 100 distinct XGBoost models by randomly generating 100 unique combinations of training and test sets. Recall that none of the test samples are used on the training step. We report on the average true acceptance rate (TAR) and the corresponding standard deviation $-\mu_{T A R}$ and $\sigma$, respectively. TAR is the rate of a correct classification of the corrosion level.

The performance of the model for the Per-Signal Scenario resulted in a $\mu_{T A R}$ of $84.3 \%$ and $\sigma$ of $0.8 \%$. Figure 19 shows a more detailed view of the results. The confusion matrix shows that the model can better discriminate between $0 \%$ and $20 \%$ corrosion levels than between $50 \%$ and $100 \%$. These results are encouraging from a practical perspective, because it is preferred to sense early stages of corrosion damage rather than discriminating at a more severe corrosion stage.

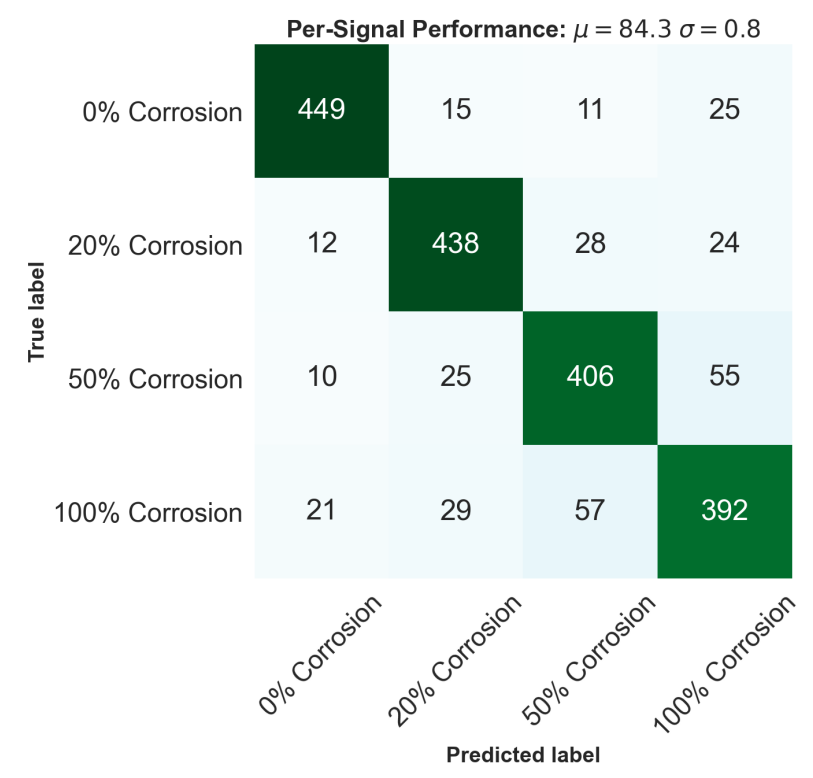

Figure 19. Confusion matrix for Per-Signal Model.

As mentioned above, average-fusion and median-fusion methods were generated for the per-scan scenario. The performance of average-fusion resulted in a $\mu_{T A R}$ of $90.8 \%$ and $\sigma$ of $2.2 \%$, while the performance for median-fusion resulted in a $\mu_{T A R}$ of $99.5 \%$ and $\sigma$ of $0.6 \%$. The ML model continues to perform better at lower level of corrosion than at severe levels. Figure 20(a) breaks down the performance at different levels 
of corrosion for the average-fusion method. Note that in contrast with the per-signal method, the per-scan average model perfectly separates the classes for lower corrosion levels from the higher corrosion levels. The best performance was observed by the median-fusion method. As shown in Figure 20(b), the median-fusion method nearly correctly classified all specimen scans. The contrasting performance between the average and median methods offer important insight about our application. The average-fusion method picks the average prediction value, which is prone to be affected by outliers due to the random texture in specimens. On the other hand, median measurements tend to perform robustly in the presence of outliers when a considerable number of the measurements still fit expected distribution. For our application, most of the transducer pairs will collect correct measurements. Therefore, selecting the median value as the final predicted value results in a more robust method and near perfect performance.

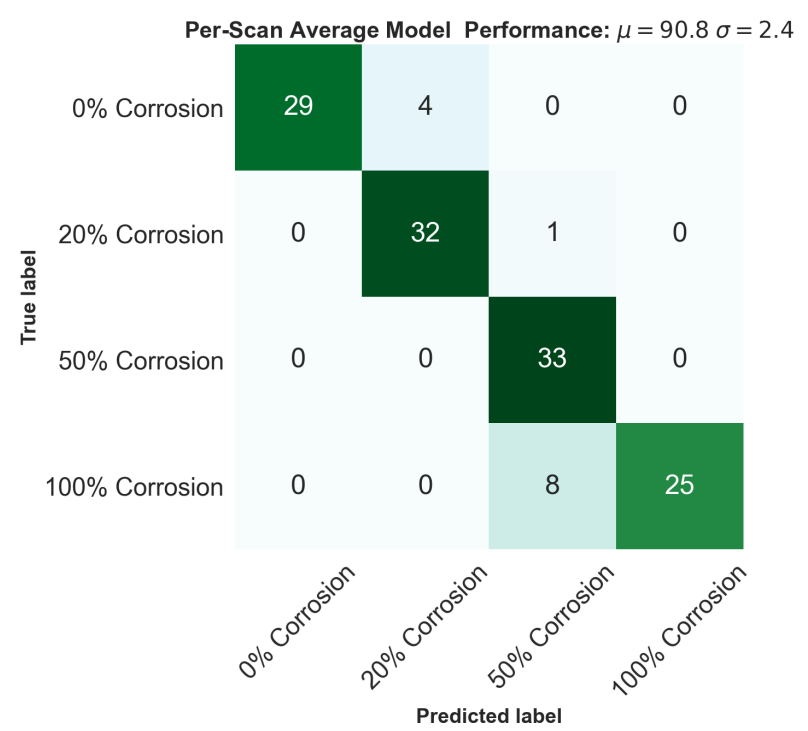

(a)

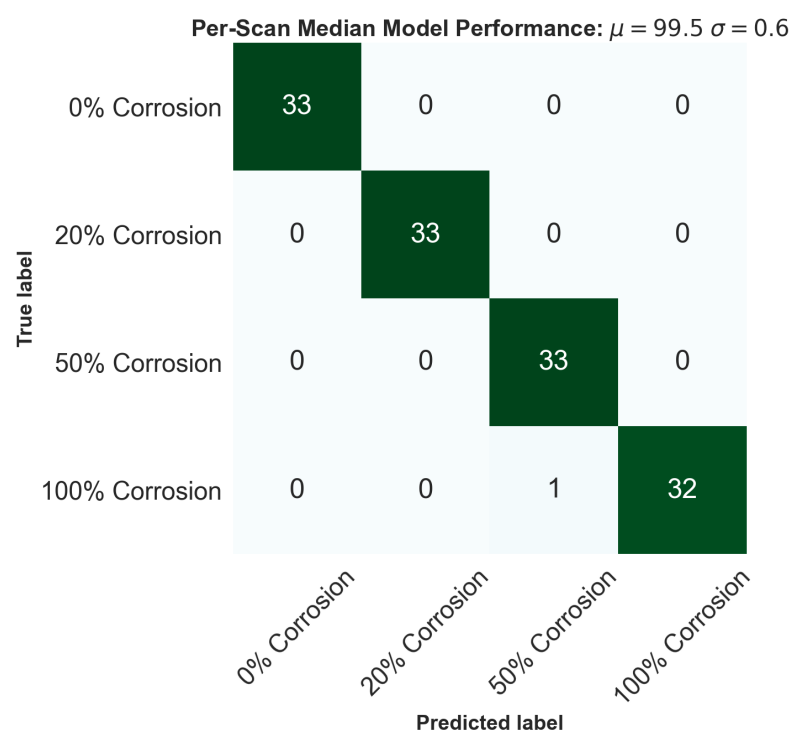

(b)

Figure 20. Confusion matrix for Per-Scan (a) Average-fusion and (b) Median-fusion methods.

Finally, Table 7 shows the overall average TAR and standard deviation results for the per-pair scenario. Individually, each transducer pair performed similarly to the broken down results in the per-signal scenario (See Figure 19). Table 7 results show that transducer position and distance between pairs did not have a model performance effect. However, for the synthetic experiments the introduced random texture was applied evenly across the specimen. Therefore, all transducers pairs were arbitrarily equally affected. In real life, specimen texture may have a bias toward certain specimen regions. For those specimens, location and spacing between transducer may be important. In addition, in our experiments, corrosion was applied uniformly around the rebar. This may not be the case for real life scenarios. Finally, new system geometries and sizes should be assessed. These factors could be explored in follow on work.

\section{CONCLUSIONS}

This project studied the capabilities of MBIR and ML algorithms in the imaging and estimation of rebar corrosion from ultrasound signals. The application was challenging due to the introduction of a steel liner 


\begin{tabular}{|c|c|c|c|c|c|}
\hline \multirow{2}{*}{ Sources } & \multicolumn{5}{|c|}{ Receivers } \\
\hline & 1 & 2 & 3 & 4 & 5 \\
\hline 1 & $84.3 \pm 0.5$ & $84.1 \pm 0.6$ & $84.1 \pm 0.5$ & $84.1 \pm 0.4$ & $84.2 \pm 0.3$ \\
\hline 2 & - & $84.2 \pm 0.2$ & $84.2 \pm 0.1$ & $84.3 \pm 0.1$ & $84.3 \pm 0.1$ \\
\hline 3 & - & - & $84.3 \pm 0.1$ & $84.2 \pm 0.1$ & $84.3 \pm 0.1$ \\
\hline 4 & - & - & - & $84.3 \pm 0.1$ & $84.2 \pm 0.1$ \\
\hline 5 & - & - & - & - & $84.2 \pm 0.1$ \\
\hline
\end{tabular}

Table 7. Per-Pair Scenario $\mu_{T A R} \pm \sigma$ performance results.

between the sensor and the concrete containing the rebar. Initially, we intended to study imaging of two rebars-located at $44.5 \mathrm{~mm}$ and $323.8 \mathrm{~mm}$ from the sensor. The attenuation generated by the steel liner precluded us from imaging the rebar at $323.8 \mathrm{~mm}$. Therefore, such specimen geometry was abandoned and we focused in a specimen with a single rebar at a depth of $44.5 \mathrm{~mm}$ with four levels of corrosion; 0\%, 20\%, $50 \%$, and $100 \%$ corrosion level. The corrosion was applied uniformly around the rebar, generating a ring in the perimeter of the rebar with different acoustic density. In order to generate a realistic synthetic dataset, we added random texture to the concrete of the synthetic specimens. This texture will mimic the variations encountered in real concrete specimens.

Our first approach was to develop an imaging technique to obtain a cross-section of the interior of the specimen. The goal was to develop an image reconstruction technique that produces an image in near-real-time (i.e., less than 2 minutes). We adapted our MBIR algorithm for the application. MBIR was able to reconstruct the specimen even under severe reverberation signals at the steel liner. The off-the-shelve MBIR technique was able to resolve the steel liner with artifacts on the periphery and the rebar at corrosion levels $0 \%, 50 \%$, and $100 \%$. The $20 \%$ corrosion ring was harder to observe in the reconstructions. Note that the $20 \%$ corrosion ring thickness is a sub-wavelength feature. Two qualitative metrics were implemented to assess MBIR performance. The quantitative results were in agreement with the qualitative assessment.

For most real-life applications, there are specimen features that are well known, which is the case of the steel liner. The interaction between the ultrasound system and steel liner can be characterized in incorporated in our MBIR physical model. We experimented with this concept and developed a new version of MBIR names MBIR-BCM. With this enhanced MBIR algorithm, we were able to cancel out the reverberation at the at the steel-concrete interface and enhance the signals coming from the interior of the specimen. The MBIR-BCM technique boosted image quality for all corrosion level and provided quantitative results that better correlated with the corrosion level. However, reconstructions from the $0 \%$ and $20 \%$ corrosion levels were difficult to separate. Note that this is not an MBIR flaw, but a physics limit placed by the nominal frequency at the ultrasound system.

Our second approach was to develop a machine learning technique to estimate corrosion level from the ultrasound signals. We developed an XGBoost model that correctly classified corrosion level for over $99 \%$ 
of the ultrasound system scans. In order for this method to be effective, the location of the rebar needs to be known. A complete solution will be an integration of MBIR-BCM with the XGBoost model. MBIR is used to image the specimen's interior to locate in space features of interest (e.g., rebar). Each feature centroid pixel index is used to extract the corresponding ultrasound truncated signals. The set of truncated signals for each unique transducer pair is ingested by the XGBoost model for prediction. Our study concluded that the median prediction value is an appropriate method to fuse the individual transducer pair predictions for a final per-scan system decision.

The application at hand needs further study. Real life specimen always bring new challenges and non-ideal conditions. Therefore, the findings of this study need corroboration on laboratory-scale specimens and field experiments. 


\section{AUTHORS BIOGRAPHIES}

Dr. Hector J. Santos-Villalobos is a Senior R\&D Staff Member, leader of the Biometrics and Identity Research Group, and manager of the Identity Science Program for the National Security Sciences Directorate at Oak Ridge National Laboratory (ORNL). He received BS and MS degrees in 2003 and 2005, respectively, from the Department of Computer and Electrical Engineering at the University of Puerto Rico-Mayag'uez. In 2010, Dr. Santos-Villalobos received a PhD from the School of Electrical and Computer Engineering at Purdue University and right after, he joined ORNL as a postdoctoral student. In 2011, he was one of the recipients of the JIST \& JEI Itek Award for an outstanding and original research publication on imaging science and engineering concerning his doctoral work. During his time at ORNL, he has worked on a variety of projects with a focus on computational imaging, machine learning, and biometrics. His research has produced several technical publications, issued patents, and research grants.

Dr. Pradeep Ramuhalli is a nuclear instrumentation and controls engineer and 4 a Distinguished R\&D Staff Member at ORNL. Prior to joining ORNL, he was a senior research scientist at Pacific Northwest National Laboratory and a faculty member in the Department of Electrical and Computer Engineering at Michigan State University. He received a PhD in electrical engineering from Iowa State University in 2002. Dr. Ramuhalli's research over the past 15 years has focused on technology developments to enable resilience and reliability in complex systems, and includes sensor design, numerical modeling, ML for condition monitoring and prognostics, inverse problems, and multisensory data fusion. His research at ORNL focuses on the development of sensors and data analysis algorithms for condition monitoring of structures and systems, prognostics algorithms to assess remaining useful life and support operational decision-making, and the application of ML to construct digital twins for use in assuring reliability of measurements and resilience of degraded systems.

\section{Abdulrahman M. Alanazi}

is a doctoral student in the School of Electrical and Computer Engineering at Purdue University. He received his BSc degree in electrical engineering with first-class honors from Tabuk University, Saudi Arabia, in 2014. He obtained his MSc degree in electrical engineering in 2016, with a focus on image deblurring and denoising, from King Abdullah University of Science and Technology, Saudi Arabia. Currently, he is pursuing his $\mathrm{PhD}$ degree with a focus on the intersection between ultrasound imaging, optimization, and ML, under the supervision of Professor Charles Bouman. 


\section{REFERENCES}

Agarwala, V. S., Reed, P. L., and Ahmad, S. (2000). Corrosion detection and monitoring - a review. In Corrosion 2000, page 19, NACE. NACE International.

Almansouri, H., Venkatakrishnan, S., Bouman, C., and Santos-Villalobos, H. (2018a). Model-based iterative reconstruction for one-sided ultrasonic nondestructive evaluation. IEEE Transactions on Computational Imaging, 5(1):150-164.

Almansouri, H., Venkatakrishnan, S., Clayton, D., Polsky, Y., Bouman, C., and Santos-Villalobos, H. (2018b). Anisotropic modeling and joint-map stitching for improved ultrasound model-based iterative reconstruction of large and thick specimens. In AIP Conference Proceedings, volume 1949 (1), page 030002. AIP Publishing LLC.

Almansouri, H., Venkatakrishnan, S., Clayton, D., Polsky, Y., Bouman, C., and Santos-Villalobos, H. (2018c). Ultrasonic model-based iterative reconstruction with spatially variant regularization for one-sided non-destructive evaluation. Electronic Imaging, 2018(15):103-1.

Barandas, M., Folgado, D., Fernandes, L., Santos, S., Abreu, M., Bota, P., Liu, H., Schultz, T., and Gamboa, H. (2020). Tsfel: Time series feature extraction library. SoftwareX, 11:100456.

Beard, M. D., Lowe, M. J. S., and Cawley, P. (2003). Ultrasonic guided waves for inspection of grouted tendons and bolts. Journal of Materials in Civil Engineering, 15(3):212-218.

Beniwal, S. and Ganguli, A. (2015). Defect detection around rebars in concrete using focused ultrasound and reverse time migration. Ultrasonics, 62:112-125.

Berndt, M. (2001). Non-Destructive Testing Methods For Geothermal Piping. Technical report, U.S. Department of Energy.

Chen, T. and Guestrin, C. (2016). Xgboost: A scalable tree boosting system. In Proceedings of the 22nd acm sigkdd international conference on knowledge discovery and data mining, pages 785-794.

Cheng, Y., Hanif, A., Chen, E., Ma, G., and Li, Z. (2018). Simulation of a novel capacitive sensor for rebar corrosion detection. Construction and Building Materials, 174:613-624.

de Alcantara Jr., N. P., da Silva, F. M., GuimarÃ£es, M. T., and Pereira, M. D. (2016). Corrosion assessment of steel bars used in reinforced concrete structures by means of eddy current testing. Sensors, $16(15)$.

Dobie, G., Pierce, S. G., and Hayward, G. (2013). The feasibility of synthetic aperture guided wave imaging to a mobile sensor platform. NDT and E International, 58(7):10-17.

Engle, B. J., Schmerr, J. L. W., and Sedov, A. (2014). Quantitative ultrasonic phased array imaging. AIP Conf. Proc., 1581(7):49.

François, R., Khan, I., and Dang, V. (2012). Impact of corrosion on mechanical properties of steel embedded in 27-year-old corroded reinforced concrete beams. Materials and Structures, 46.

François, R., Laurens, S., and Deby, F. (2018a). Corrosion and its consequences for reinforced concrete structures. Elsevier. 
François, R., Laurens, S., and Deby, F. (2018b). Corrosion and Its Consequences for Reinforced Concrete Structures. Elsevier.

Guarneri, G. A., Pipa, D. R., Junior, F. N., de Arruda, L. V. R., and Zibetti, M. V. W. (2015). A sparse reconstruction algorithm for ultrasonic images in nondestructive testing. Sensors, 15(4):9324-9343.

Haldorsen, J., Johnson, D., Plona, T., Sinha, B., Valero, H., and Winkler, K. (2006). Borehole acoustic waves. Oilfield Review, 18(1):34-43.

Hoegh, K. and Khazanovich, L. (2015). Extended synthetic aperture focusing technique for ultrasonic imaging of concrete. NDT and E International, 74:33-42.

Li, D., Zhang, S., Yang, W., and Zhang, W. (2014). Corrosion Monitoring and Evaluation of Reinforced Concrete Structures Utilizing the Ultrasonic Guided Wave Technique. International Journal of Distributed Sensor Networks, 10(2):827130.

Li, M. and Hayward, G. (2011). Ultrasound Nondestructive Evaluation (NDE) imaging with transducer arrays and adaptive processing. Sensors, 12(12):42-54.

Maack, S., Thunell, B., and Wiggenhauser, H. (2015). Study of Special Challenges for Standard NDT-Methods on Nuclear Structures. In International Symposium Non-Destructive Testing in Civil Engineering (NDTCE 2015). NDT.net.

Ozkan, E., Vishnevsky, V., and Goksel, O. (2017). Inverse problem of ultrasound beamforming with sparsity constraints and regularization. IEEE Transactions on Ultrasonics, Ferroelectrics, and Frequency Control.

Pieper, D., Donnell, K., Ghasr, M., and Kinzel, E. (2014). Integration of microwave and thermographic ndt methods for corrosion detection. AIP Conference Proceedings, 1581.

Ramadan, S., Gaillet, L., Tessier, C., and Idrissi, H. (2008). Assessment of the Stress Corrosion Cracking in a Chloride Medium of Cables Used in Prestressed Concrete Structures by the Acoustic Emission Technique. Measurement Science E Technology, 19(11):115702 (9 pp.).

Ramuhalli, P., Griffin, J. W., Meyer, R. M., Pitman, S. G., Fricke, J. M., Dahl, M. E., Prowant, M. S., Kafentzis, T. A., Coble, J. B., and Roosendaal, T. J. (2012). Nondestructive Examination (NDE) Detection and Characterization of Degradation Precursors, Technical Progress Report for FY 2012. United States Department of Energy Office of the Assistant Secretary for Nuclear Energy.

Schickert, M., Krause, M., and Müller, W. (2003). Ultrasonic imaging of concrete elements using reconstruction by synthetic aperture focusing technique. Journal of Materials in Civil Engineering, 15(3):235-246.

Shao, Z., Shi, L., Shao, Z., and Cai, J. (2011). Design and application of a small size SAFT imaging system for concrete structure. Review of Scientific Instruments, 82(7):073708.

Singh, R., Raj, B., Mudali, U. K., and Singh, P. (2019). Non-Destructive Evaluation of Corrosion and Corrosion-assisted Cracking. Wiley.

Sriramadasu, R. C., Banerjee, S., and Lu, Y. (2019). Detection and assessment of pitting corrosion in rebars using scattering of ultrasonic guided waves. NDT $\mathcal{F}$ E International, 101:53-61. 
Stepinski, T. (2007). An implementation of synthetic aperture focusing technique in frequency domain. ieee transactions on ultrasonics, ferroelectrics, and frequency control, 54(7).

Tang, Q. and Yu, T. (2017). Finite element simulation of ultrasonic waves in corroded reinforced concrete for early-stage corrosion detection, volume 10169 of SPIE Smart Structures and Materials + Nondestructive Evaluation and Health Monitoring. SPIE.

Thibault, J.-B., Sauer, K. D., Bouman, C. A., and Hsieh, J. (2007). A three-dimensional statistical approach to improved image quality for multislice helical ct. Medical physics, 34(11):4526-4544.

Treeby, B. E., Budisky, J., Wise, E. S., Jaros, J., and Cox, B. (2018). Rapid calculation of acoustic fields from arbitrary continuous-wave sources. The Journal of the Acoustical Society of America, 143(1):529-537.

Treeby, B. E. and Cox, B. T. (2010). k-wave: Matlab toolbox for the simulation and reconstruction of photoacoustic wave fields. Journal of biomedical optics, 15(2):021314.

Treeby, B. E., Jaros, J., Rendell, A. P., and Cox, B. (2012). Modeling nonlinear ultrasound propagation in heterogeneous media with power law absorption using ak-space pseudospectral method. The Journal of the Acoustical Society of America, 131(6):4324-4336.

Treeby, B. E., Jaros, J., Rohrbach, D., and Cox, B. (2014). Modelling elastic wave propagation using the k-wave matlab toolbox. In 2014 IEEE international ultrasonics symposium, pages 146-149. IEEE.

Tuysuzoglu, A., Kracht, J. M., Cleveland, R. O., Cs etin, M., and Karl, W. C. (2012). Sparsity driven ultrasound imaging a. The Journal of the Acoustical Society of America, 131(2):1271-1281.

Villalobos, S., Johnson, S. R., and Scott, D. (2019). Inspection of Concrete Behind Steel Liners Using Ultrasonic Methods. In NDE in Nuclear 2019. NDT.net.

Watanabe, T., Trang, H. T. H., Harada, K., and Hashimoto, C. (2014). Evaluation of corrosion-induced crack and rebar corrosion by ultrasonic testing. Construction and Building Materials, 67:197-201.

Wu, H., Chen, J., Wu, S., Jin, H., and Yang, K. (2015). A model-based regularized inverse method for ultrasonic B-scan image reconstruction. Measurement Science and Technology, 26(10):105401.

Yeih, W. and Huang, R. (1998). Detection of the corrosion damage in reinforced concrete members by ultrasonic testing. Cement and Concrete Research, 28(7):1071-1083.

Zemanek, J., Glenn, E. E., Norton, L. J., and Caldwell, R. L. (1970). Formation evaluation by inspection with the borehole televiewer. Geophysics, 35(2):254-269.

Zou, X., Schmitt, T., Perloff, D., Wu, N., Yu, T.-Y., and Wang, X. (2015). Nondestructive corrosion detection using fiber optic photoacoustic ultrasound generator. Measurement, 62:74-80. 